NATIONAL LABORATORY

\title{
MULTIVARIATE HIGH ORDER STATISTICS OF MEASUREMENTS OF THE TEMPORAL EVOUTION OF FISSION CHAIN-REACTIONS
}

\author{
J. K. MATINGG LY
}

MARC H 2001

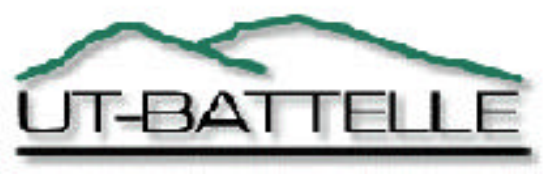




\section{DISCLAIMER}

This report was prepared as an account of work sponsored by an agency of the United States government. Neither the United States Government nor any agency thereof, nor any of their employees, makes any warranty, express or implied, or assumes any legal liability or responsibility for the accuracy, completeness, or usefulness of any information, apparatus, product, or process disclosed, or represents that its use would not infringe privately owned rights. Reference herein to any specific commercial product, process, or service by trade name, trademark, manufacturer, or otherwise, does not necessarily constitute or imply its endorsement, recommendation, or favoring by the United States Government or any agency thereof. The views and opinions of authors expressed herein do not necessarily state or reflect those of the United States Government or any agency thereof. 
ORNL/TM-2001/45

\title{
Multivariate High Order Statistics of Measurements of the Temporal Evolution of Fission Chain-Reactions
}

\author{
J. K. Mattingly
}

March 2001

Prepared by

OAK RIDGE NATIONAL LABORATORY

Oak Ridge, Tennessee 37831-6004

managed by

UT-BATTELLE, LLC.

for the

U.S. DEPARTMENT OF ENERGY

under contract DE-AC05-00OR22725 


\section{CONTENTS}

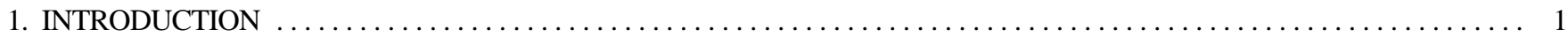

2. THEORETICAL MODELS OF STATISTICS OF MEASUREMENTS

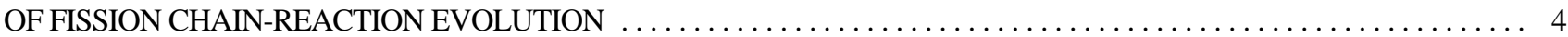

3. GENERAL PROPERTIES OF HIGH ORDER STATISTICS AND THEIR POTENTIAL UTILITY $\ldots \ldots \ldots \ldots \ldots \ldots \ldots 21$

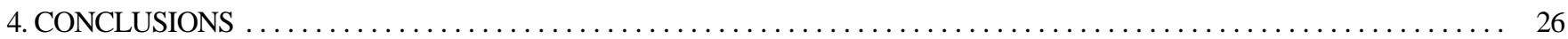

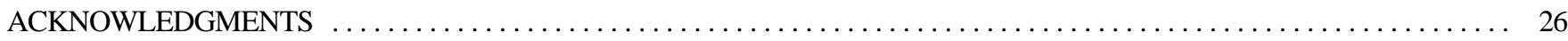

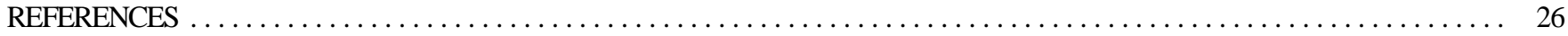




\section{LIST OF F IGURES}

Figure 1 One possible chain-reaction that can occur during an active measurement of a fissile system;

closed circles denote observable events including detector counts and detected source emissions,

open circles denote unobservable induced fission events, and each line indicates a neutron

Figure 2 Diagram representing chain-reaction shown in Fig. 1; each line now indicates a series of "chain-related" neutrons, and the label associated with each line indicates the number of neutrons available to sustain that branch in the chain-reaction

Figure 3 Diagram representing a chain-reaction terminating in one detector count

Figure 4 Diagram representing chain-reactions terminating in two correlated detector counts; the notation indicating each process denotes the neutron economy of the chain-reaction

Figure 5 Diagram representing chain-reactions terminating in three correlated detector counts; the notation indicating each process again denotes the neutron economy of the chain-reaction

Figure 6 Diagrammatic representation of all chain-reactions terminating in from one to five detector counts; diagrams in the leftmost column are minimal because they are formed from one symbol, and all subsequent diagrams are composites formed by concatenation of minimal symbols. Diagrams beginning with "(1-1)' are not shown here because they are identical to the diagrams Shown right-concatenated to the symbol for " 1 ". 


\title{
Multivariate High Order Statistics of Measurements of the Temporal Evolution of Fission Chain-Reactions
}

\author{
J. K. Mattingly \\ Oak Ridge National Laboratory, Instrumentation and Controls Division \\ Oak Ridge, Tennessee 37831-6004
}

\begin{abstract}
The development of high order statistical analyses applied to measurements of the temporal evolution of fission chain-reactions is described. These statistics are derived via application of Bayes' rule to conditional probabilities describing a sequence of events in a fissile system beginning with the initiation of a chain-reaction by source neutrons and ending with counting events in a collection of neutron-sensitive detectors. Two types of initiating neutron sources are considered: (1) a directly observable source introduced by the experimenter (active initiation), and (2) a source that is intrinsic to the system and is not directly observable (passive initiation). The resulting statistics describe the temporal distribution of the population of prompt neutrons in terms of the timedelays between members of a collection (an $\mathrm{n}$-tuplet) of correlated detector counts, that, in turn, may be collectively correlated with a detected active source neutron emission. These developments are a unification and extension of Rossi- $\alpha$, pulsed neutron, and neutron noise methods, each of which measure the temporal distribution of pairs of correlated events, to produce a method that measures the temporal distribution of $\mathrm{n}$-tuplets of correlated counts of arbitrary dimension $\mathrm{n}$. In general the technique should expand present capabilities in the analysis of neutron counting measurements.
\end{abstract}

\section{INTRODUCTION}

The present work focuses on multivariate statistics derived from counting measurements performed on fissile systems driven by a neutron source that is introduced by the experimenter or is intrinsic or inherent to the system. Measurements performed using an introduced source to initiate fission chain-reactions are described as "active," and measurements that rely solely upon an intrinsic source to initiate fission are described as "passive." It should be noted that the introduction of an active source does not necessarily preclude the presence of an intrinsic source; in many applications of interest, the two will be present simultaneously. Instead, the active source is introduced to provide a means of observing the time of initiation of some chain-reactions. In particular, it is assumed that the active neutron source is directly observable in that it is intsrumented in such a way that individual neutron emission events may be counted.

For example, one candidate active source is the ${ }^{252} \mathrm{Cf}$ ionization chamber. This device detects the fragments of individual spontaneous fissions of ${ }^{252} \mathrm{C}$ nuclei such that the ionization chamber signal, when acquired by pulse processing instrumentation, registers the time of neutron emission events. Note that the number of neutrons emitted during a given spontaneous fission is not observable by this instrument.[1] Another candidate active source is the associated-particle neutron generator (APNG) that accelerates a projectile ion (typically a deuteron) into a target (typically deuterium, as in a D-D generator, or tritium, as in a D-T generator) to produce a single neutron per collision. The neutron emission event is registered by detection of the collision product ion (a.k.a., the associated-particle, a ${ }^{3} \mathrm{He}$ nucleus from a D-D reaction or an ${ }^{4} \mathrm{He}$ nucleus from a D-T reaction).[2] In the subsequent development, an observable neutron source is treated generically as a device that registers the time of individual neutron emission events, that is in general capable of emitting multiple neutrons per event, but that is incapable of registering the number of neutrons emitted during a single event. Note that an APNG is simply a special case of this generic active source because only a single neutron is emitted per projectile-target collision.

Passive sources, on the other hand, are intrinsic to the fissile system and are not directly instrumented like active sources. Consequently, neither the time of a particular neutron emission event nor the number of neutrons emitted during the event is in general observable. For example, ${ }^{240} \mathrm{Pu}$ and ${ }^{242} \mathrm{Pu}$ are spontaneous fission sources that are typically present in any 
system containing ${ }^{239} \mathrm{Pu} ;{ }^{9} \mathrm{Be}$ in the presence of plutonium, by virtue of plutonium $\alpha$-decay, can form a Pu-Be source that emits a single neutron per $(\alpha, n)$ reaction in ${ }^{9} \mathrm{Be}$. Other examples of intrinsic neutron sources are ${ }^{242} \mathrm{Cm}$ and ${ }^{244} \mathrm{Cm}$ which appear as fission products in burnt $\mathrm{UO}_{2}$ (and other ${ }^{235} \mathrm{U}$-containing) nuclear fuels. Measurements of systems containing such intrinsic sources may be performed passively by relying solely upon these sources to initiate fission chain-reactions, or they may be performed actively such that fission chain-reactions are initiated by both the introduced source and intrinsic sources. The choice of active versus passive measurement depends upon the application and upon practical considerations like the relative strengths of the active and intrinsic sources.

Finally, it is important to note that every fissile system contains an inherent delayed neutron source. Technically, delayed neutrons are correlated progeny of a fission chain-reaction just as are prompt neutrons. However, generations of delayed neutrons typically reproduce much more slowly than do generations of prompt neutrons. Consequently, relative to the time-scale of a prompt neutron "fission-chain", delayed neutrons typically behave as a source inherent to the system that is uncorrelated with the prompt progeny of the chain-reaction.

The response of the fissile system to the preceding sources is observed by a collection of neutron-sensitive detectors whose signals are acquired by pulse processing instrumentation such that the time of each neutron detection event is registered. These detectors in principle may operate via any number of physical processes; however, to keep the subsequent development simple, it is assumed that the detectors operate via neutron absorption such that a detection event is always a terminus in a chain-reaction. In other words, the detector removes the detected neutron from the population and does not return any further neutrons to the population. Consequently, the following developments do not immediately encompass scatter detectors (e.g., plastic scintillators) or fission detectors. However, modifications to the theory required to address these kinds of detectors have been described in the context of previous theories and can be readily extended to apply to the present work.[3,4]

Previous developments including the Rossi- $\alpha$, pulsed neutron, and neutron noise methods measure the distribution of two-way coincidence between pairs of events including detector counts and, if an active source is employed, detected source emissions. Central to (although sometimes implicit in) these methods is the concept of the covariance

$$
\left.R_{12}(\mathrm{~J}) \quad C_{12}(\mathrm{~J}) \& \overline{x_{1}} \overline{x_{2}} \quad E\left[x_{1}\left(t_{1}\right) x_{2}\left(t_{2}\right)\right]\right|_{J}{ }_{t_{2} \& t_{1}} \& \overline{x_{1}} \overline{x_{2}}
$$

between a pair of signals $\left(x_{1}, x_{2}\right)$ as a function of the time-delay $\mathrm{J}$ between an event in channel 1 and an event in channel 2. Above, both " $E[\quad]$ " and " $=$ ", denote the expected value, or mean, such that $C_{12}(\mathrm{~J})$ is the mean total coincidence rate between signals $x_{1}$ and $x_{2}$ when channel 2 is delayed by $\mathrm{J}$ relative to channel 1 . The mean rate of "accidental" coincidence, i.e., the rate of uncorrelated coincident events is the product of mean count rates $\overline{x_{1}} \overline{x_{2}}$. Consequently, $R_{12}(\mathrm{~J})$ is the rate of "real"coincidence, i.e., the rate of events coincident at a relative delay of $\mathrm{J}$ that are correlated between channels 1 and 2.

In the context of the Rossi- $\alpha$ method, both signals are acquired from radiation detectors such that $R_{12}(\mathrm{~J})$ is the distribution of correlated pairs of counts over the relative delay $\mathrm{J}$ between the counts in the $\left(x_{1}, x_{2}\right)$ pair. [5-8] In the context of a pulsed neutron measurement, channel 1 is a "trigger" signal acquired from an instrumented active source and channel 2 is a radiation detector signal. Consequently in this context $R_{12}(\mathrm{~J})$ is the distribution of "source-correlated" detector counts over the delay $\mathrm{J}$ following an initial source emission.[9] An active neutron noise measurement employing two or more radiation detectors is essentially a simultaneous Rossi- $\alpha$ and pulsed neutron measurement in that it measures the distribution of twoway coincidence between all pairs of signals; a passive neutron noise measurement is essentially the same as a Rossi- $\alpha$ measurement. The only distinction between neutron noise measurements and Rossi- $\alpha /$ pulsed neutron measurements is the way in which the distributions of pairs are analyzed. Neutron noise measurements often employ digital signal processing methods to acquire each distribution of pairs in the frequency- (i.e., Fourier- or Laplace-) domain:

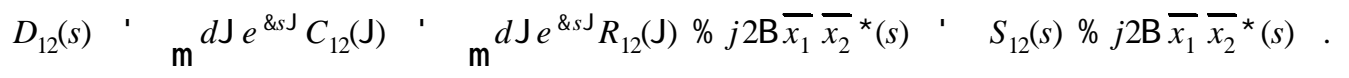

\footnotetext{
${ }^{1}$ A Rossi- $\alpha$ measurement may use only a single detector such that $x_{1}$ and $x_{2}$ are actually the same signal.[5-8]
} 
The spectral density $D_{12}\left(s^{\prime} j \mathrm{~T}\right)$ is simply the decomposition of the total coincidence rate distribution $C_{12}(\mathrm{~J})$ into harmonic components $e^{\delta \mathrm{j} T J}{ }^{2}$ The principal reasons (among others) for the use of the frequency-domain representation are (1) to permit digital acquisition and analysis of analog signals (e.g., current-mode signals from an active source and/or detectors) and (2) to facilitate rapid processing of the signals by taking advantage of fast Fourier transform (FFT) algorithms. $[10]$ However, because in this application the transformation is isomorphic (if the signals are processed correctly), the covariance between signals $R_{12}(\mathrm{~J})$ can always be synthesized by inverse transformation of the harmonic spectrum $S_{12}(s)$.[13-15] Consequently, Rossi- $\alpha$, pulsed neutron, and neutron noise measurements can be grouped as a single method that measures the distribution of real two-way coincidence between pairs of observed events.

The present work extends this method to measure distributions of $n$-way coincidence between an arbitrary number $n$ of observed events. ${ }^{4}$ Central to the method is the notion of a total poly-coincidence distribution

$$
C_{12, n}\left(\mathrm{~J}_{1}, \mathrm{~J}_{2}, \stackrel{\nu}{ }, \mathrm{J}_{n \& 1}\right), E\left[x_{1}\left(t_{1}\right) x_{2}\left(t_{2}\right), x_{n}\left(t_{n}\right)\right] \begin{array}{ll}
J_{1}, & t_{2} \& t_{1} \\
\vdots & t_{3} \& t_{1} \\
! & \\
t_{n} \& t_{1}
\end{array},
$$

from which a corresponding poly-covariance can be obtained by subtracting the relevant accidental poly-coincidence. The form of the accidental poly-coincidence depends upon the order $n$ of the total distribution; for example the accidental polycoincidence for a third-order statistic is

$$
\overline{x_{1}} R_{23}\left(\mathrm{~J}_{2} \& \mathrm{~J}_{1}\right) \% \overline{x_{2}} R_{13}\left(\mathrm{~J}_{2}\right) \% \overline{x_{3}} R_{12}\left(\mathrm{~J}_{1}\right) \% \overline{x_{1}} \overline{x_{2}} \overline{x_{3}}
$$

while for a fourth-order statistic it is

$$
\begin{gathered}
\overline{x_{1}} R_{234}\left(\mathrm{~J}_{2} \& \mathrm{~J}_{1}, \mathrm{~J}_{3} \& \mathrm{~J}_{1}\right) \% \overline{x_{2}} R_{134}\left(\mathrm{~J}_{2}, \mathrm{~J}_{3}\right) \% \overline{x_{3}} R_{124}\left(\mathrm{~J}_{1}, \mathrm{~J}_{3}\right) \% \overline{x_{4}} R_{123}\left(\mathrm{~J}_{1}, \mathrm{~J}_{2}\right) \% \\
R_{12}\left(\mathrm{~J}_{1}\right) R_{34}\left(\mathrm{~J}_{3} \& \mathrm{~J}_{2}\right) \% R_{13}\left(\mathrm{~J}_{2}\right) R_{24}\left(\mathrm{~J}_{3} \& \mathrm{~J}_{1}\right) \% R_{14}\left(\mathrm{~J}_{3}\right) R_{23}\left(\mathrm{~J}_{2} \& \mathrm{~J}_{1}\right) \% \\
\overline{x_{1}} \overline{x_{2}} R_{34}\left(\mathrm{~J}_{3} \& \mathrm{~J}_{2}\right) \% \overline{x_{1}} \overline{x_{3}} R_{24}\left(\mathrm{~J}_{3} \& \mathrm{~J}_{1}\right) \% \overline{x_{1}} \overline{x_{4}} R_{23}\left(\mathrm{~J}_{2} \& \mathrm{~J}_{1}\right) \% \overline{x_{2}} \overline{x_{3}} R_{14}\left(\mathrm{~J}_{3}\right) \% \overline{x_{2}} \overline{x_{4}} R_{13}\left(\mathrm{~J}_{2}\right) \% \overline{x_{3}} \overline{x_{4}} R_{12}\left(\mathrm{~J}_{1}\right) \% \\
\overline{x_{1}} \overline{x_{2}} \overline{x_{3}} \overline{x_{4}} .
\end{gathered}
$$

${ }^{2}$ Elsewhere this quantity has been referred to as a cross-power spectral density (CPSD), a power spectral density (PSD), or a power spectrum.[10] Subsequently the Laplace transform of a covariance $R$ (not a total coincidence distribution $C$ ) shall be simply termed a spectrum $S$; the context of use will clearly distinguish this harmonic spectrum from an energy spectrum, e.g., a fission neutron spectrum. The Laplace-domain representation shall be used exclusive of the Fourier representation; the latter is simply a special case (i.e., $s^{\prime} j \mathrm{~T}$ ) of the former.

${ }^{3}$ It is interesting to note that some neutron noise measurements in fact pre-date the development of the FFT.[11,12]

${ }^{4}$ This is not a new concept in counting measurements; $n$-way coincidence gates have been available for some time.[16] 
Observe that they simply represent the different ways that $n$ uncorrelated events can be $n$-way coincident. ${ }^{5}$ A second central concept in the method is that of a poly-spectrum

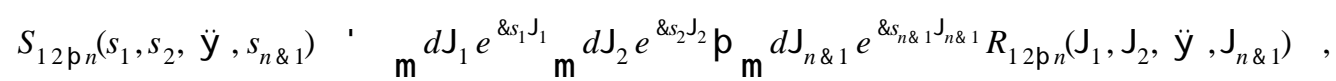

that is simply an $n$-dimensional harmonic decomposition of the poly-covariance. Because of the dual nature of convolution in the time-domain $(\mathrm{J})$ and multiplication in the frequency-domain $(s)$, it will prove easier to generalize the subsequent models of high order statistics to arbitrary order $n$ in the frequency-domain.

These concepts have been "borrowed" from the broader field of high order statistical analysis to be applied to measurements of the temporal evolution of fission chain-reactions.[17] Research into this extension is presented in the remainder of the paper as follows. Section II describes a probabilistic representation of the sequence of events that can give rise to a family of $n$ correlated neutron counts. Application of Bayes' rule to conditional probabilities relating each event in the sequence to its predecessors yields the joint probability of the sequence of events. From such joint probabilities the poly-covariance for each specific sequence of events is immediately obtained. Inductive reasoning is subsequently applied to a diagrammatic representation of sequences of events to yield a "prescription" to obtain statistics of arbitrary order. Section III describes properties of the derived high order statistics and emphasizes their progressively higher sensitivity to reactivity. Most of the observations made here transcend the limitations of the simple point model used to derive the statistics in Section II. Several applications taking advantage of the properties of high order statistics are also suggested in Section III. Concluding remarks are made at the close of the paper in Section IV.

\section{THEORETICAL MODELS OF STATISTICS OF MEASUREMENTS OF FISSION CHAIN-REACTION EVOLUTION}

Figure 1 diagrammatically depicts one possible chain-reaction that can occur during an active measurement of a fissile system. In the figure observable events, i.e., the active source emission $x_{0}\left(t_{0}\right)$ (the initiating event) and each of the detection events $x_{1}\left(t_{1}\right)$ through $x_{6}\left(t_{6}\right)$ (the terminal events), are represented by closed circles. The induced fission events (intermediate events) are in general unobservable and are represented by open circles . Each line connecting events in the sequence represents a single neutron. Observe that the terminal events are collectively "chain-related," or correlated, to the initiating event by the depicted sequence of intermediate events. Consider that only the branching points in the chain-reaction determine the nature of the correlation between the detection events and the initiating source emission. The intermediate induced fissions along any single branch simply serve to sustain the chain-reaction to a detection event. Consequently, this chain-reaction can be represented by the simpler equivalent diagram shown in Fig. 2. In this figure, each line connecting events in the sequence represents a series of chain-related neutrons (and not a single neutron as in Fig. 1). The label (e.g. “ $<$, " $<\& 1 "$, , $<\& 2$ ", etc.) associated with each line denotes the number of neutrons available to sustain that branch in the chain-reaction.

The joint probability of the sequence of events represented by the diagram prescribes the nature of the correlation between the initiating and terminal events. By using Bayes' rule, such joint probabilities can be generated from the marginal probability of the initiating event, the conditional probability for each intermediate event given the preceding initiating or intermediate events, and the conditional probability for each terminal event given the preceding initiating or intermediate events.[18] For example, the joint probability of a sequence of events $(A, B, C, D, E)$ is just

${ }^{5}$ Although the binomial expansion of an $n$ th-order central moment of the form $E\left[\left(x_{1} \& \overline{x_{1}}\right)\left(x_{2} \& \overline{x_{2}}\right)\right.$, $\left.\left(x_{n} \& \overline{x_{n}}\right)\right]$ yields the accidental poly-coincidence for second- and third-order statistics, this is not true of higher order statistics. In general, for an $n$ th-order statistic, the form of the accidental poly-coincidence can be obtained by partitioning the set $\left(x_{1}, x_{2},{ }^{\vee}, x_{n}\right.$ : into all permutations of two, three, ${ }^{\vee}$, and $n$ non-empty subsets. The beginning of this progression is illustrated in (5). However, because the accidental poly-coincidence can always be obtained from lower order statistics, and because it arises due to uncorrelated coincident events such that it is of no real interest, there will no subsequent mention of accidental poly-coincidence. Our real interest lies in the distribution of correlated events, the poly-covariance. 


$$
p_{A B C D E}(A, B, C, D, E) \quad ' \quad p_{E^{*} D C B A}\left(E^{*} D, C, B, A\right) \bigotimes_{D^{*} C B A}\left(D^{*} C, B, A\right) \bigotimes_{C^{*} B A}\left(C^{*} B, A\right) \bigotimes_{B^{*} A}\left(B^{*} A\right) \bigotimes_{A}(A)
$$

where $A$ is the initial event, and $B, C, D$, and $E$ are either intermediate or terminal events. This is the nature of the subsequent developments that describe first- through third-order statistics. Diagrammatic representations like that depicted in Fig. 2 are eventually used to generalize these statistics to arbitrary order $n$.

The statistics of interest are the poly-covariances between a collection of $n$ signals, which may include a signal acquired from an instrumented active source. Once the joint probability $p_{12, n}\left(t_{1}, t_{2},{ }^{v}, t_{n}\right)$ of a sequence of $n$ chain-related observable events has been determined, the corresponding poly-covariance is simply

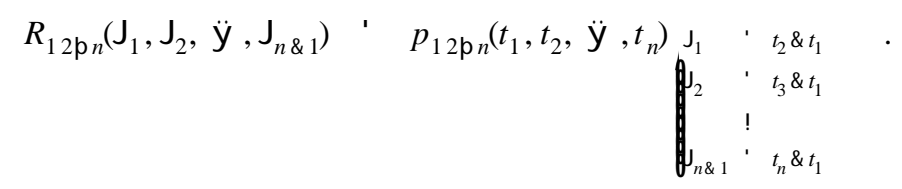

In other words, the poly-covariance is the expected rate of poly-coincidence between $n$ correlated events when the second through $n$th events are respectively delayed by $J_{1}$ through $J_{n \& 1}$ relative to the first event.

In order to model the probability of a series of chain-related prompt neutrons, the point reactor impulse response

$$
\left.\left.p_{*}\left(t^{\prime} * t\right) d t \quad \frac{e^{\varepsilon^{\prime \prime}(t)}}{7} u(t) \& t\right) d t\right)
$$

is used to approximately represent the probable number of neutrons present at time $t^{\prime}$ per neutron injected into the fissile system at time $t$.[19] Above, $u$ represents the causal function (the Heaviside step function), " denotes the decay constant for prompt-neutron-induced fission chain-reactions (the Rossi- $\alpha$ ), and 7 denotes the prompt neutron generation time, i.e., the mean time between generations of prompt neutrons. ${ }^{6}$ Subsequently, given that <neutrons were injected at $t$, the probability that a detection event $\mathrm{M}$ (a count) will occur at $t_{\mathrm{M}}$ is

$$
p_{\mathrm{M} *}\left(t_{\mathrm{M}} * t, \triangleleft d t_{\mathrm{M}} \quad, \quad, \mathrm{M} p *\left(t_{\mathrm{M}} * t\right) d t_{\mathrm{M}}<1,{ }_{\mathrm{M}} \frac{e^{\varepsilon "\left(t_{\mathrm{M}} \& t\right)}}{7} u\left(t_{\mathrm{M}} \& t\right) d t_{\mathrm{M}}<\right.
$$

where ${ }_{M}$ denotes the efficiency of detector M, i.e., the probability per neutron of a count in detector M. Furthermore, given that the mean time between prompt-neutron-induced fissions is $<7$, where $\overline{<}$ is the mean number of prompt neutrons emitted per induced fission, the probable number of induced fissions at time $t^{\prime}$ per neutron injected at time $t$ is

$$
q *\left(t^{\prime} * t\right) d t \quad, \quad \frac{e^{\varepsilon^{\prime \prime}\left(t^{\prime} \& t\right)}}{\overline{<}} u\left(t^{\prime} \& t\right) d t^{\prime}
$$

Subsequently, given that <neutrons were injected at $t$, the probability that an induced fission $\mathrm{F}$ will occur at $t_{\mathrm{F}}$ and emit $<_{F}$ neutrons is

$$
q_{\mathrm{F} *}\left(t_{\mathrm{F}},<_{\mathrm{F}} * t, \triangleleft d t_{\mathrm{F}} \quad ' \quad p_{\mathrm{F}}\left(<_{\mathrm{F}}\right) q *\left(t_{\mathrm{F}} * t\right) d t_{\mathrm{F}}<' \quad p_{\mathrm{F}}\left(<_{\mathrm{F}}\right) \frac{e^{\varepsilon^{\prime \prime}\left(t_{\mathrm{F}} \& t\right)}}{<7} u\left(t_{\mathrm{F}} \& t\right) d t_{\mathrm{F}}<\right.
$$

where $p_{\mathrm{F}}\left(<_{\mathrm{F}}\right)$ denotes the probability that $<_{\mathrm{F}}$ prompt neutrons will emerge from the induced fission $\mathrm{F}$.

Observe that the joint probability of multiple detection events chain-related to a single emission can be expressed as a product of conditional probabilities using Bayes' rule. For example, the probability of two detection events $\mathrm{M}$ and $\mathrm{M}^{\prime}$ chainrelated to the same neutron emission is

${ }^{6}$ Recall that prompt reactivity $\left.D^{\prime}\right) k / k^{\prime} \& " 7$, where $k$ denotes the prompt multiplication factor. 


$$
\begin{gathered}
p_{\mathrm{M})}{ }^{*}\left(t_{\mathrm{M}}^{\prime}, t_{\mathrm{M}}^{*} t, \triangleleft d t_{\mathrm{M}}^{\prime} d t_{\mathrm{M}}, p_{\mathrm{M}) * \mathrm{M}}\left(t_{\mathrm{M}}^{\prime} * t_{\mathrm{M}}, t, \triangleleft d t_{\mathrm{M}}^{\prime} \bigotimes_{\mathrm{M} *}\left(t_{\mathrm{M}} * t, \triangleleft d t_{\mathrm{M}}\right.\right.\right. \\
,_{\mathrm{M}} \frac{e^{\varepsilon^{\prime \prime}\left(t_{\mathrm{M}}^{\prime} \& t\right)}}{7} u\left(t_{\mathrm{M}}^{\prime} \& t\right) d t_{\mathrm{M}}^{\prime}(<\& 1) @_{\mathrm{M}} \frac{e^{\varepsilon^{\prime \prime}\left(t_{\mathrm{M}} \& t\right)}}{7} u\left(t_{\mathrm{M}} \& t\right) d t_{\mathrm{M}}<,
\end{gathered}
$$

because the first detection event $M$ leaves only $<\& 1$ neutrons to induce the second detection event $M$ ). In general, the probability of $n$ detection events correlated to a single emission is

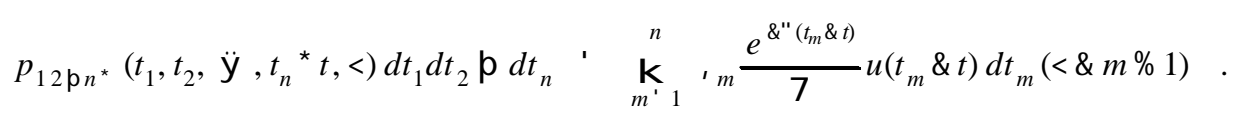

Similarly, multiple fissions may be induced by a single emission. Finally, multiple detection events and induced fissions may be chain-related through not only one but instead a sequence of emission events. For example, in Figs. 1 and 2 the detection events $x_{1}\left(t_{1}\right), x_{2}\left(t_{2}\right), x_{3}\left(t_{3}\right)$, and $x_{4}\left(t_{4}\right)$ are all collectively chain-related to the source emission $x_{0}\left(t_{0}\right)$ through the sequence of induced fissions $x(t)$ and $x^{\prime}\left(t^{\prime}\right)$. The joint probability of such a sequence of events is more complicated than that of the sequence represented in (14). This will become evident during the subsequent developments.

All fission chain-reactions are initiated by some spontaneous source emission, and during any given measurement a number of independent sources may be present. Let channel " 0 " denote an instrumented active source signal such that the probability of a detected source emission at $t_{0}$ that injects $<_{0}$ neutrons is

$$
q_{0}\left(t_{0}, \varangle_{0}\right) d t_{0} \quad q_{0}\left(t_{0}\right) d t_{0} p_{0}\left(\varangle_{0}\right) \quad ' \quad p_{0}\left(\varangle_{0}\right),{ }_{0} Q_{0} d t_{0}
$$

where $p_{0}\left(\varsigma_{0}\right)$ is the probability that $<_{0}$ neutrons will emerge from an active source emission,,${ }_{0}$ denotes the efficiency of the active source, i.e., the probability that a source emission will be detected, and $Q_{0}$ denotes the active source emission rate. ${ }^{7}$ Recall that it is assumed that the active source is in general capable of producing multiple neutrons per emission, but that only the emission event itself, and not the number of neutrons produced, can be detected. Consequently, the active source has an observable mean emission rate of

$$
\overline{x_{0}},{ }_{0} Q_{0}
$$

However, unobserved source emissions may also initiate fission-chains, and the probability that an undetected active source emission will inject $<$ neutrons at $t_{0}$ is ${ }^{8}$

$$
q_{0}^{(}\left(t_{0},<_{0}\right) d t_{0} \quad, \quad p_{0}\left(<_{0}\right)\left(1 \&,{ }_{0}\right) Q_{0} d t_{0}
$$

Finally, intrinsic neutron sources, which are collectively denoted by "G", may also initiate fission-chains such that the probability of an intrinsic source emission at $t_{\mathrm{G}}$ that injects $<_{\mathrm{G}}$ neutrons is

$$
q_{\mathrm{G}}\left(t_{\mathrm{G}},<_{\mathrm{G}}\right) d t_{\mathrm{G}} \quad p_{\mathrm{G}}\left(<_{\mathrm{G}}\right) Q_{\mathrm{G}} d t_{\mathrm{G}}
$$

Each of the preceding independent sources serve to induce fission in the system such that the marginal probability of an induced fission at $t$ that produces $<$ neutrons is

${ }^{7}$ In general, the source emission rate may decay with time as, e.g., $Q_{0} \exp \left(\& 8_{0} t_{0}\right)$. However, because the duration of a typical measurement is short compared to the decay time of a typical active source (e.g., the half-life of ${ }^{252} \mathrm{Cf}$ is roughly 2.6 years), decay of the source will be neglected.

${ }^{8}$ Note that a non-instrumented active source can be represented by letting , 60 , and a passive measurement can be represented by letting $Q_{0} 60$. 


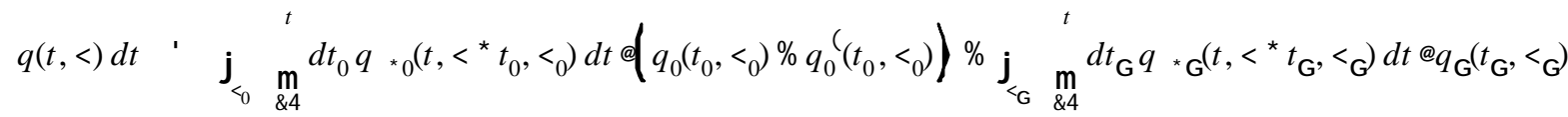

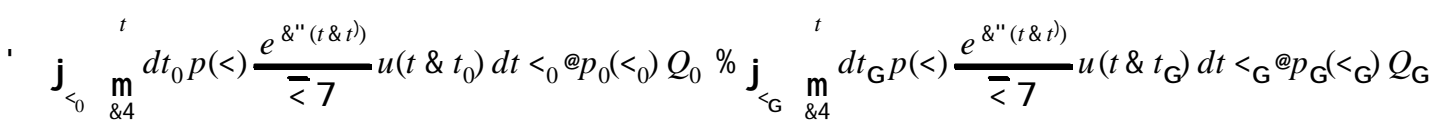

$$
\begin{aligned}
& \text { ' } p\left(\triangleleft \frac{\left(\overline{\gamma_{0}} Q_{0} \% \overline{\varepsilon_{\mathrm{G}}} Q_{\mathrm{G}}\right)}{\overline{<}^{\prime \prime} 7} d t \quad, \quad p(\triangleleft Q d t\right.
\end{aligned}
$$

These are of course simplified representations of the probabilities governing fission chain-reactions; however, most of the subsequent observations are more general than the simple point models used to describe the statistics.

\subsection{FIRST-ORDER STATISTICS}

First-order statistics describe the mean rate of individual neutron counting events, which may in turn be correlated to detected active source emissions. Observe that the joint probability of a detection event chain-related to a detected active source emission is simply

$$
\begin{aligned}
& p_{01}\left(t_{0}, t_{1}\right) d t_{0} d t_{1} \quad \mathrm{j}_{\delta} p_{1 *_{0}}\left(t_{1} * t_{0}, \succ_{0}\right) d t_{1} @_{0}\left(t_{0}, \succ_{0}\right) d t_{0} \\
& \text { ' } \mathrm{j}_{\delta},{ }_{1} \frac{e^{\delta^{\prime \prime}\left(t_{1} \& t_{0}\right)}}{7} u\left(t_{1} \& t_{0}\right) d t_{1}<\Phi_{0}\left(\zeta_{0}\right),{ }_{0} Q_{0} d t_{0} \quad, \quad,{ }_{1} \frac{\bar{\delta}_{{ }_{0}} Q_{0}}{7} e^{\varepsilon^{\prime \prime}\left(t_{1} \& t_{0}\right)} u\left(t_{1} \& t_{0}\right) d t_{0} d t_{1} .
\end{aligned}
$$

The (almost trivial) diagram for this joint probability is shown in Fig. 3. Subsequently, the covariance between the detector and active source signals is

$$
\left.R_{01}(\mathrm{~J}) \quad p_{01}\left(t_{0}, t_{1}\right)\right|_{J} \cdot t_{1} \&_{0}, \quad, \bar{\zeta}_{1} Q_{0} Q_{0} e^{\alpha^{\prime \prime J}} u(\mathrm{~J})
$$

This is the quantity measured by a pulsed neutron experiment; it is the distribution of detector counts chain-related to some detected active source emission over the delay J between initial source emission and the subsequent count.[9] Neutronnoise analysis experiments often represent this distribution as a harmonic spectrum[10]

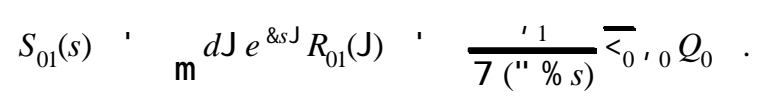

Subsequently, the marginal probability of a count chain-related to a detected active source emission is

$$
p_{1}^{0}\left(t_{1}\right) d t_{1} \quad \operatorname{m}_{\delta 4}^{t_{1}} d t_{0} p_{01}\left(t_{0}, t_{1}\right) d t_{1} \quad{ }^{\prime} \quad \mathrm{j}_{\delta} \operatorname{m}_{\delta 4}^{t_{1}} d t_{0} p_{1 * 0}\left(t_{1} * t_{0}, \delta_{0}\right) d t_{1} @_{0}\left(t_{0}, \delta\right)
$$

However, some detector counts may instead be chain-related to an undetected active source emission with probability

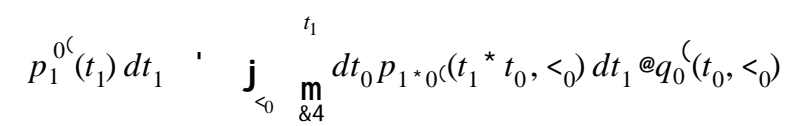


or to an intrinsic source emission with probability

$$
p_{1}^{\mathrm{G}}\left(t_{1}\right) d t_{1} \quad \mathrm{j}_{\mathcal{G}} \operatorname{m}_{\& A}^{t_{1}} d t_{\mathrm{G}} p_{1 *_{\mathrm{G}}}\left(t_{1} * t_{\mathrm{G}},<_{\mathrm{G}}\right) d t_{1} @ \mathrm{G}_{\mathrm{G}}\left(t_{\mathrm{G}},<_{\mathrm{G}}\right)
$$

such that the total marginal probability of a detector count is

$$
p_{1}\left(t_{1}\right) d t_{1} \quad\left(p_{1}^{0}\left(t_{1}\right) \% p_{1}^{0^{\prime}}\left(t_{1}\right) \% p_{1}^{\mathrm{G}}\left(t_{1}\right) \% B_{1}\right) d t_{1}
$$

where $B_{1}$ denotes the background count rate observed in the absence of any source. Consequently,

$$
p_{1}\left(t_{1}\right) d t_{1} \quad, \quad \frac{\left(\overline{\zeta_{0}} Q_{0} \% \overline{\varepsilon_{\mathrm{G}}} Q_{\mathrm{G}}\right)}{" 7} d t_{1} \% B_{1} d t_{1} \quad, \quad,{ }_{1} \overline{<} Q d t_{1} \% B_{1} d t_{1}
$$

such that the mean detector count rate is

$$
\bar{x}_{1}, p_{1}, \quad, \frac{\left(\overline{\delta_{0}} Q_{0} \% \overline{\varepsilon_{\mathrm{G}}} Q_{\mathrm{G}}\right)}{" 7} \% B_{1} .
$$

In accordance with (26), the count rate is comprised of components arising from counts chain-related to each contributing source described in (15), (17), and (18):

$$
\begin{gathered}
\overline{x_{1}}, \overline{x_{1}^{0}} \% \overline{x_{1}^{0}} \% \overline{x_{1}^{\mathrm{G}}} \% B_{1} \\
\mathrm{~m}^{d \mathrm{~J} R_{01}(\mathrm{~J}) \%} \mathrm{~m}^{d \mathrm{~J} R_{0}{ }_{1}(\mathrm{~J}) \%} \mathrm{~m}^{d \mathrm{~J} R_{\mathrm{G} 1}(\mathrm{~J}) \% B_{1}} \\
S_{01}(0) \% S_{0(1}(0) \% S_{\mathrm{G} 1}(0) \% B_{1}
\end{gathered}
$$

where $R_{0}{ }_{1}$ and $R_{\mathrm{G} 1}$ are the analogs to $R_{01}$ respectively obtained by substituting the sources " 0 " " and " $\mathrm{G}$ " for the source "0" in (20). Subsequently, a "source-conditional" covariance $R_{1 *_{0}}$ may be defined

$$
R_{01}(\mathrm{~J}) \quad R_{1 * 0}(\mathrm{~J}) \overline{x_{0}} \quad,\left.\quad p_{1 *_{0}}\left(t_{1} *_{t_{0}}\right)\right|_{\mathrm{J}}{ }^{\prime} t_{1} \& t_{0} @_{0}
$$

such that the corresponding conditional mean is

$$
\overline{x_{1 * 0}} \quad, \quad \mathrm{r}^{d \mathrm{~J} R_{1 *_{0}}(\mathrm{~J})}
$$

This is the mean number of counts correlated to a detected source emission per detected source emission. Consequently, the fraction of all counts that are chain-related to detected active source emissions is

$$
\frac{\overline{x_{1 * 0}}}{\overline{x_{1}} / \overline{x_{0}}}, \frac{\mathrm{r}^{d \mathrm{~J} R_{01}(\mathrm{~J})}}{\overline{x_{1}}}, \frac{S_{01}(0)}{\overline{x_{1}}}, \frac{,_{0}}{1 \% \frac{\overline{\bar{\varepsilon}_{\mathrm{G}}} Q_{\mathrm{G}}}{\overline{\varepsilon_{0}} Q_{0}} \% \frac{B_{1}}{{ }_{1} \overline{\delta_{0}} Q_{0} / " 7}} .
$$

${ }^{9}$ The quantities $R_{0^{\prime} 1}$ and $R_{\mathrm{G} 1}$ (and consequently $\overline{x_{1}^{0}}, \overline{x_{1}^{\mathrm{G}}}, S_{0^{\prime} 1}$, and $S_{\mathrm{G} 1}$ ) are entirely conceptual; they cannot be directly measured since the sources " 0 " " and "G" are not directly observable. 
In other words, because the covariance between the active source and detector signals is the distribution of counts chainrelated to the active source, it can be used in conjunction with the detector count rate to distinguish the count rate arising from detected active source emissions from that arising from unobservable sources.

\subsection{SECOND-ORDER STATISTICS}

Second-order statistics describe the mean rate of correlated pairs of neutron counting events, which may in turn be collectively correlated to detected active source emissions. Two unique processes can give rise to a correlated pair of counts chain-related to an initial source emission as depicted in Fig. 4. The notation used to denote each process indicates the neutron "economy" of the process. The process labeled "2" begins with the injection of source neutrons which each initiate at least two separate branches in the chain-reaction that in turn terminate in at least two counts. The process labeled "(1-

1) +2 " begins with the injection of source neutrons that successfully initiate only one branch in the chain-reaction that in turn terminates in at least one induced fission (hence the notation "(1-1)"). This induced fission subsequently injects at least two more neutrons (hence the notation " +2 ") into the system that initiate at least two separate branches in the chain-reaction that in turn terminate in at least two counts. These two processes are mutually exclusive such that

$$
p_{012}\left(t_{0}, t_{1}, t_{2}\right) d t_{0} d t_{1} d t_{2} \quad\left({ }_{2} p_{012}\left(t_{0}, t_{1}, t_{2}\right) \%_{(1 \& l) \%} p_{012}\left(t_{0}, t_{1}, t_{2}\right)\right) d t_{0} d t_{1} d t_{2}
$$

The joint probability of process 2 is

$$
\begin{aligned}
& \left.{ }_{2} p_{012}\left(t_{0}, t_{1}, t_{2}\right) \quad \mathrm{j}_{\delta} p_{2 * 10}\left(t_{2} * t_{1}, t_{0},\right\rangle_{0}\right) \bigotimes_{1 *_{0}}\left(t_{1} * t_{0},>_{0}\right) \bigotimes_{0}\left(t_{0},>_{0}\right) \\
& \mathrm{j}_{\delta},_{2} \frac{e^{\&^{\prime \prime}\left(t_{2} \& t_{0}\right)}}{7} u\left(t_{2} \& t_{0}\right)(\delta \& 1) \bigotimes_{1} \frac{e^{\&^{\prime \prime}\left(t_{1} \& t_{0}\right)}}{7} u\left(t_{1} \& t_{0}\right)<\bigotimes_{0}\left(\delta_{0}\right){ }_{0} Q_{0} \\
& ,,_{1},{ }_{2} \frac{\overline{\delta_{0}\left(\delta_{0} \& 1\right)},{ }_{0} Q_{0}}{7^{2}} e^{\&^{\prime \prime}\left(t_{1} \& t_{0}\right)} e^{\& \prime\left(t_{2} \& t_{0}\right)} u\left(t_{1} \& t_{0}\right) u\left(t_{2} \& t_{0}\right)
\end{aligned}
$$

and is invariant over a permutation in the sequence of counts $\left(x_{1}, x_{2}\right)$. That is, the probability of process 2 is the same regardless of which detector count occurs first. On the other hand,

$$
{ }_{(1 \& 1) \%} p_{012}\left(t_{0}, t_{1}, t_{2}\right) \quad\left\{\left\{\begin{array}{ll}
(1 \& 1) \% p_{012}\left(t_{0}, t_{1}, t_{2} \$ t_{1}\right), & t_{2} \$ t_{1} \\
(1 \& 1) \% p_{012}\left(t_{0}, t_{1}, t_{2}<t_{1}\right), & t_{2}<t_{1}
\end{array},\right.\right.
$$

i.e., the probability of the pair of counts $\left(x_{1}, x_{2}\right)$ under process $(1-1)+2$ varies depending upon the order of the two counts. This is so because the induced fission at $t$ must occur sometime between the source emission at $t_{0}$ and the first of the two counts. Consequently,

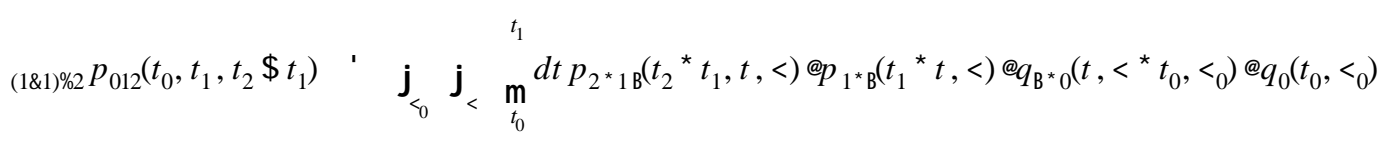

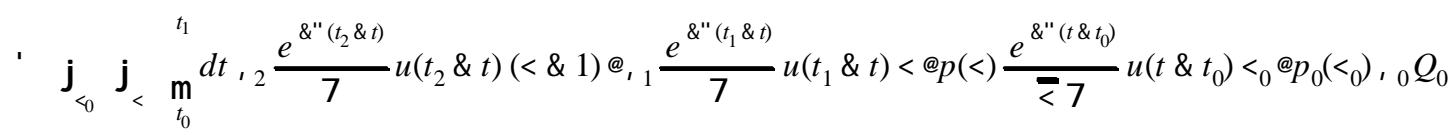

$$
\begin{aligned}
& \frac{\prime_{1}{ }_{2}}{7^{2}} \frac{\overline{<(<\& 1)} \bar{\delta},_{0} Q_{0}}{\overline{<}^{\prime \prime} 7} e^{\& \prime\left(t_{1} \& t_{0}\right)} u\left(t_{1} \& t_{0}\right)\left(e^{\& \prime\left(t_{2} \& t_{1}\right)} u\left(t_{2} \& t_{1}\right) \& e^{\& \prime\left(t_{2} \& t_{0}\right)} u\left(t_{2} \& t_{0}\right)\right),
\end{aligned}
$$


while

$$
\begin{aligned}
& (1 \& 1) \% p_{012}\left(t_{0}, t_{1}, t_{2}<t_{1}\right) \quad \text { ' } \mathrm{j}_{\delta} \mathrm{j}_{<} \mathrm{m}_{t_{0}}^{t_{2}} d t p_{1 *_{2} \mathrm{~B}}\left(t_{1} * t_{2}, t, \triangleleft \bigotimes_{2 * \mathrm{~B}}\left(t_{2} * t, \triangleleft \bigotimes_{\mathrm{B}{ }_{0}}\left(t,<* t_{0},<_{0}\right) \bigotimes_{0}\left(t_{0},>_{0}\right)\right.\right.
\end{aligned}
$$

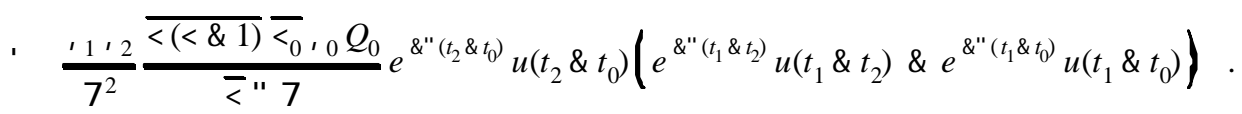

Note that a reversal in the order of the counts results in probabilities that are simply "mirror images" of one another. This is so because reversing the order of the counts simply reverses the relative delays $\mathrm{J}_{1}{ }^{\prime} t_{1} \& t_{0}$ and $\mathrm{J}_{2}{ }^{\prime} t_{2} \& t_{0}$ between each count and the initial source emission such that ${ }^{10}$

$$
\begin{aligned}
& \left(x_{0}, x_{1}, x_{2}\right): t_{2}>t_{1}>t_{0}: \mathrm{J}_{2}>\mathrm{J}_{1}>0, \\
& \left(x_{0}, x_{2}, x_{1}\right): t_{1}>t_{2}>t_{0}: \mathrm{J}_{1}>J_{2}>0 .
\end{aligned}
$$

In other words, the joint probability of two counts collectively chain-related to a source emission is reflexive over a permutation in the order of counts, as is the bicovariance $R_{012}$ between the source and detector signals:

$$
p_{012}\left(t_{0}, t_{1}, t_{2}\right) \quad ' \quad p_{021}\left(t_{0}, t_{2}, t_{1}\right): R_{012}\left(\mathrm{~J}_{1}, \mathrm{~J}_{2}\right) \quad ' \quad R_{021}\left(\mathrm{~J}_{2}, \mathrm{~J}_{1}\right)
$$

Consequently,

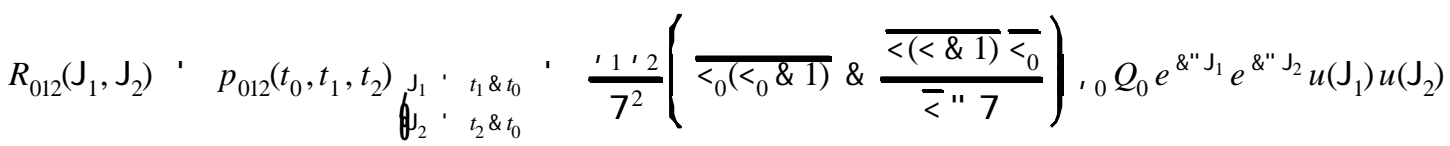

$$
\begin{aligned}
& \% \frac{\prime_{1}, 2}{7^{2}} \frac{\overline{\langle(<\& 1)} \bar{\zeta}_{{ }^{\prime}{ }_{0} Q_{0}}}{\overline{<}^{\prime \prime} 7}\left(e^{\& " J_{2}} u\left(J_{2} \& J_{1}\right) \% e^{\& J^{\prime \prime} J_{1}} u\left(J_{1} \& J_{2}\right)\right)
\end{aligned}
$$

is the distribution of correlated pairs of counts collectively chain-related to some detected active source emission over the relative delays $J_{1}$ and $J_{2}$ between each count in the pair and the initial source emission. The bispectrum between the source and detector signals is just the two-dimensional Laplace transform of the bicovariance:

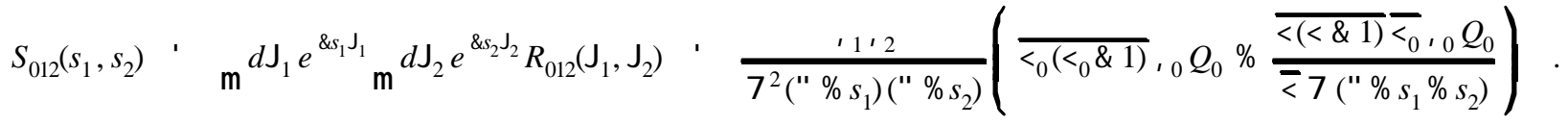

Observe that the bispectrum clearly distinguishes between the two processes that produce source-correlated pairs.

Recognize that correlated pairs of counts can arise from any of the three sources " 0 ", " 0 (", and "G", such that

$$
\begin{aligned}
& p_{12}\left(t_{1}, t_{2}\right) \quad ' \quad p_{12}^{0}\left(t_{1}, t_{2}\right) \% p_{12}^{0^{\prime}}\left(t_{1}, t_{2}\right) \% p_{12}^{\mathrm{G}}\left(t_{1}, t_{2}\right) \\
& \min t_{1}, t_{2} \quad \min t_{1}, t_{2} \quad \min t_{1}, t_{2}
\end{aligned}
$$

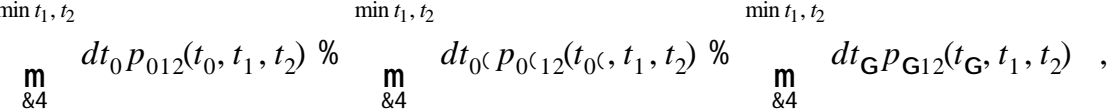

${ }^{10}$ There is no reason to consider permutations under which the source emission does not occur first; the probability of a chain-related correlated pair of counts under such a permutation is zero because the source emission must initiate the chain-reaction. 
where $p_{012}$ and $p_{\mathrm{G} 12}$ are the analogs to $p_{012}$ respectively obtained by substituting the sources " 0 " " and " $\mathrm{G}$ " for the source "0" in (34), (36), and (37). Subsequently, the covariance between two detector signals

$$
\begin{aligned}
& R_{12}(\mathrm{~J}) \text { ' }\left.\quad p_{12}\left(t_{1}, t_{2}\right)\right|_{\mathrm{J}} \quad t_{2} \& t_{1}
\end{aligned}
$$

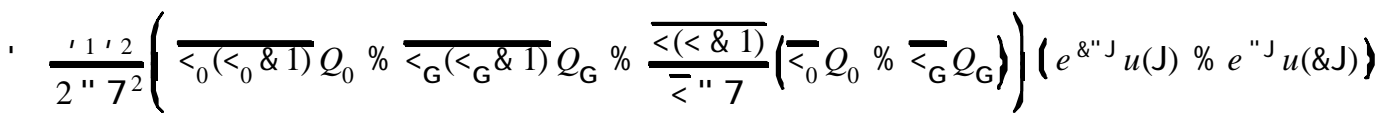

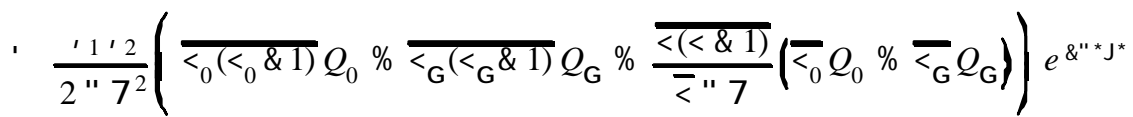

is the distribution of correlated pairs of counts over the delay $\mathrm{J}$ between the two counts in the pair. This is the quantity measured by a Rossi- $\alpha$ experiment.[5-8]

Observe that the covariance between detectors is comprised of components arising from each of the three sources

$$
\begin{aligned}
& \left.R_{12} \mathrm{~J}\right)^{\prime} \quad R_{12}^{0}(\mathrm{~J}) \% R_{12}^{0}(\mathrm{~J}) \% R_{12}^{\mathrm{G}}(\mathrm{J}){ }^{\prime} \quad \mathrm{m}^{d \mathrm{~J}_{1}} R_{012}\left(\mathrm{~J}_{1}, \mathrm{~J}_{1} \% \mathrm{~J}\right) \% \mathrm{~m}^{d \mathrm{~J}_{1}} R_{0^{(}{ }_{12}}\left(\mathrm{~J}_{1}, \mathrm{~J}_{1} \% \mathrm{~J}\right) \% \mathrm{~m} d \mathrm{~J}_{1} R_{\mathrm{G}_{12}}\left(\mathrm{~J}_{1}, \mathrm{~J}_{1} \% \mathrm{~J}\right) \\
& \mathrm{m}^{d \mathrm{~J}_{2} R_{012}\left(\mathrm{~J}_{2} \& \mathrm{~J}, \mathrm{~J}_{2}\right) \% \mathrm{~m}_{2} \mathrm{~J}_{2} R_{0}{ }_{12}\left(\mathrm{~J}_{2} \& \mathrm{~J}, \mathrm{~J}_{2}\right) \%_{\mathrm{m}} d \mathrm{~J}_{2} R_{\mathrm{G}_{12}}\left(\mathrm{~J}_{2} \& \mathrm{~J}, \mathrm{~J}_{2}\right)}
\end{aligned}
$$

Subsequently, the spectrum between detectors is[10]

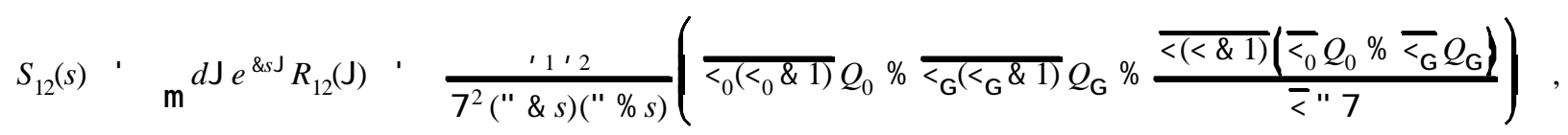

that, in accordance with (44), is comprised of contributions arising from each of the three sources

$$
\begin{gathered}
S_{12}(s) \quad S_{12}^{0}(s) \% S_{12}^{0^{l}}(s) \% S_{12}^{\mathrm{G}}(s) \\
S_{012}(\& s, s) \% S_{0^{\prime}{ }_{12}}(\& s, s) \% S_{\mathrm{G} 12}(\& s, s) .
\end{gathered}
$$

Finally, note that one can define a source-conditional covariance $R_{12 *_{0}}$ between detectors

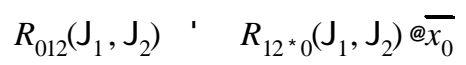

and a corresponding conditional mean $\overline{\left(x_{1} x_{2}\right)_{* 0}}$ such that

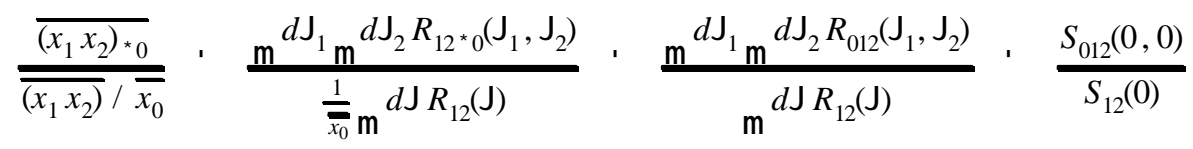

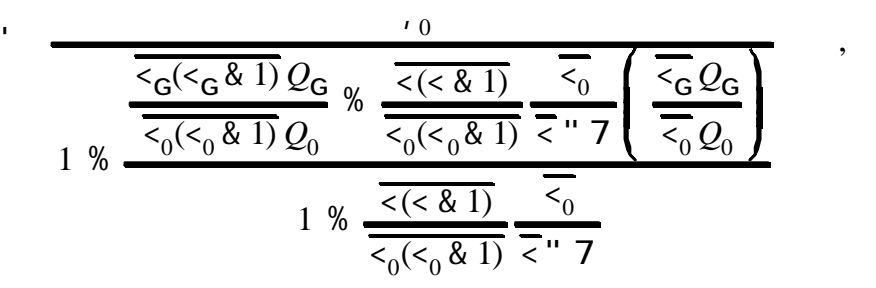


where $\overline{\left(x_{1} x_{2}\right)_{*_{0}}}$ denotes the number of correlated pairs chain-related to a detected source emission per detected source emission and $\overline{\left(x_{1} x_{2}\right)} / \overline{x_{0}}$ denotes the total number of correlated pairs per detected source emission. Consequently, (48) is the fraction of all correlated pairs that are chain-related to a detected active source emission. So the bicovariance between the source and detector signals can be used in conjunction with the covariance between detector signals to distinguish the correlated pairs rate arising from detected active source emissions from that arising from unobservable sources.

\subsection{THIRD-ORDER STATISTICS}

Third-order statistics describe the mean rate of correlated triplets of neutron counting events, which may in turn be collectively correlated to detected active source emissions. Four processes can give rise to a correlated triplet of counts chain-related to an initial source emission, and these processes are illustrated in Fig. 5. Again the notation used to denote each process indicates the neutron economy of the process. Process " 3 " is initiated by the injection of at least three source neutrons and terminates in at least three chain-related counts. Process "(2-1)+2" can occur if only two source neutrons successfully initiate separate branches in the fission-chain; one branch in this chain-reaction induces a fission (hence "(2-1)") releasing at least two neutrons that initiate two additional branches in the chain-reaction (hence "+2"). Processes "(1-1)+3" and "(1-1)+(2-1)+2" are similar to processes " 3 " and "(2-1)+2", respectively, except that each can be initiated by a single source neutron that eventually leads to an intermediate induced fission (hence "(1-1)"). These processes are mutually exclusive such that

$$
\begin{aligned}
p_{0123}\left(t_{0}, t_{1}, t_{2}, t_{3}\right) d t_{0} d t_{1} d t_{2} d t_{3} \quad & \left({ }_{3} p_{0123}\left(t_{0}, t_{1}, t_{2}, t_{3}\right) \%_{(2 \& 1) \%} p_{0123}\left(t_{0}, t_{1}, t_{2}, t_{3}\right)\right. \\
& \left.\%_{(1 \& 1) \%} p_{0123}\left(t_{0}, t_{1}, t_{2}, t_{3}\right) \%_{(1 \& 1) \%(2 \& 1) \%} p_{0123}\left(t_{0}, t_{1}, t_{2}, t_{3}\right)\right) d t_{0} d t_{1} d t_{2} d t_{3} .
\end{aligned}
$$

The joint probability of process 3 is

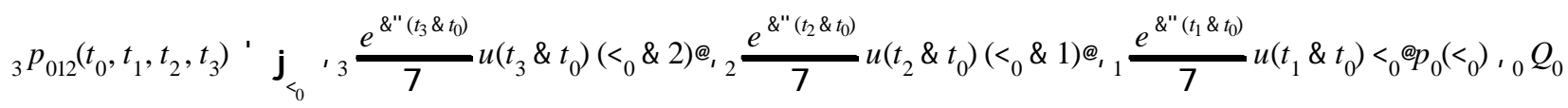

$$
\begin{aligned}
& \text { ' , , }{ }_{1}{ }_{2}{ }_{2},{ }_{3} \frac{\overline{\delta(\delta \& 1)\left(\delta_{0} \& 2\right)}{ }_{0} Q_{0}}{7^{3}} e^{\varepsilon^{\prime \prime}\left(t_{1} \& t_{0}\right)} e^{\varepsilon^{\prime \prime}\left(t_{2} \& t_{0}\right)} e^{\varepsilon^{\prime \prime}\left(t_{3} \& t_{0}\right)} u\left(t_{1} \& t_{0}\right) u\left(t_{2} \& t_{0}\right) u\left(t_{3} \& t_{0}\right)
\end{aligned}
$$

Only process 3 is invariant over permutations in the order of the three detector counts. The joint probability of the remaining processes will initially be derived for the sequence of detector counts $\left(x_{1}, x_{2}, x_{3}\right)$ occurring in exactly that order, i.e., for $t_{1} \# t_{2} \# t_{3}$. The other permutations can be subsequently derived using simple permutation relations characteristic of the joint probability (49).

The joint probability of process (2-1)+2 has three components corresponding to the three mutually exclusive conditions: (1) counts $x_{2}$ and $x_{3}$ are chain-related to the induced fission $x$; (2) counts $x_{1}$ and $x_{3}$ are chain-related to the induced fission $x$; and (3) counts $x_{1}$ and $x_{2}$ are chain-related to the induced fission $x$. Consequently, 


$$
\begin{aligned}
& (2 \& 1) \% p_{0123}\left(t_{0}, t_{1}, t_{2}>t_{1}, t_{3}>t_{2}\right) \quad '
\end{aligned}
$$

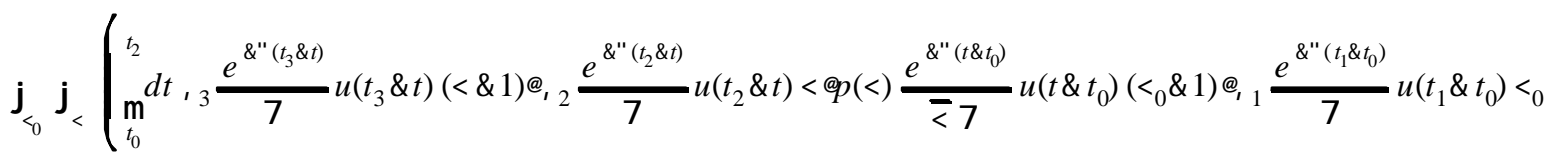

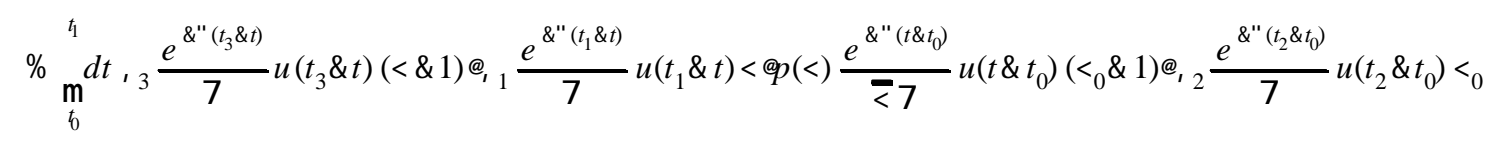

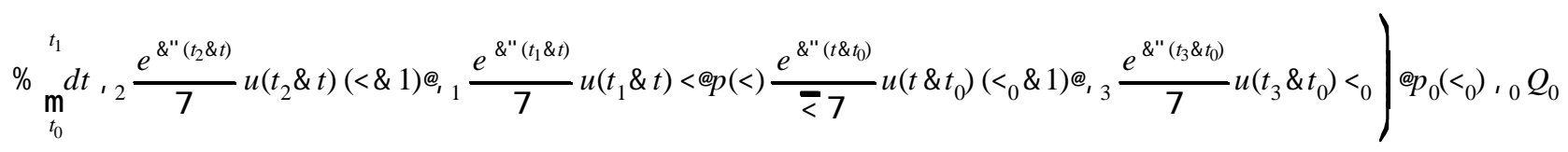

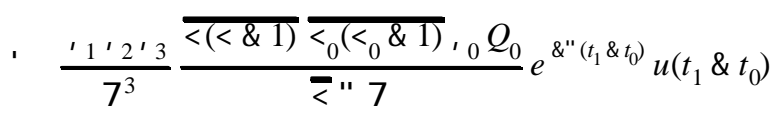

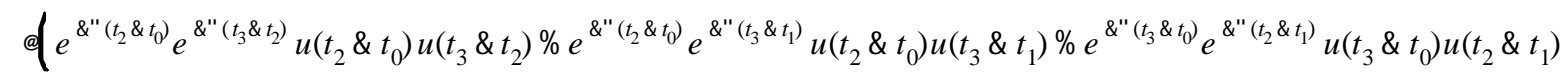

$$
\begin{aligned}
& \left.\& 3 e^{\varepsilon^{\prime \prime}\left(t_{2} \& t_{0}\right)} e^{\&^{\prime \prime}\left(t_{3} \& t_{0}\right)} u\left(t_{2} \& t_{0}\right) u\left(t_{3} \& t_{0}\right)\right) .
\end{aligned}
$$

Process (1-1)+3 is similar to process 3 except that the induced fission at $t$ must occur between the source emission at $t_{0}$ and the first count at $t_{1}$, such that

$$
\begin{aligned}
& (1 \& 1) \% p_{0123}\left(t_{0}, t_{1}, t_{2}>t_{1}, t_{3}>t_{2}\right)
\end{aligned}
$$

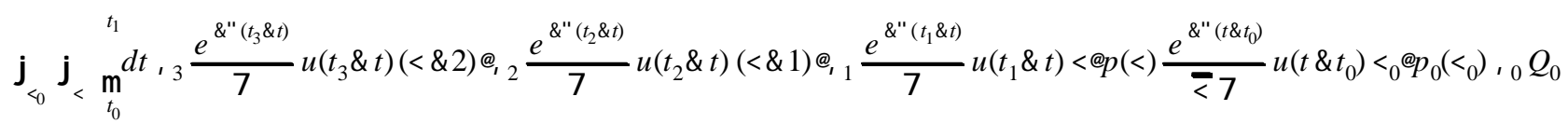

$$
\begin{aligned}
& \frac{\prime_{1}{ }_{2}{ }_{2}{ }_{3}}{27^{3}} \frac{\overline{\varangle<\& 1)(<\& 2)} \bar{\delta}_{{ }_{0}} Q_{0}}{\overline{<}^{\prime \prime} 7} e^{\&{ }^{\prime \prime}\left(t_{1} \& t_{0}\right)} u\left(t_{1} \& t_{0}\right) \\
& \left.\oint e^{\varepsilon^{\prime \prime}\left(t_{2} \& t_{1}\right)} e^{\&^{\prime \prime}\left(t_{3} \& t_{1}\right)} u\left(t_{2} \& t_{1}\right) u\left(t_{3} \& t_{1}\right) \& e^{\delta^{\prime \prime}\left(t_{2} \varangle t_{0}\right)} e^{\&^{\prime \prime}\left(t_{3} \varangle t_{0}\right)} u\left(t_{2} \& t_{0}\right) u\left(t_{3} \& t_{0}\right)\right) .
\end{aligned}
$$

Finally, process $(1-1)+(2-1)+2$ is similar to process $(2-1)+2$ except that again the induced fission $x(t)$ must occur between $x_{0}\left(t_{0}\right)$ and $x_{1}\left(t_{1}\right)$. Therefore, 


$$
\begin{aligned}
& (1 \& 1) \%(2 \& 1) \% p_{0123}\left(t_{0}, t_{1}, t_{2}>t_{1}, t_{3}>t_{2}\right) \quad '
\end{aligned}
$$

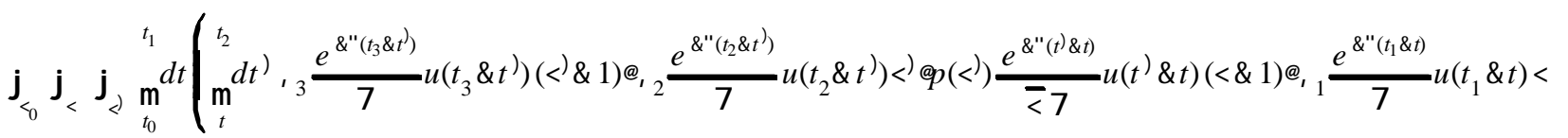

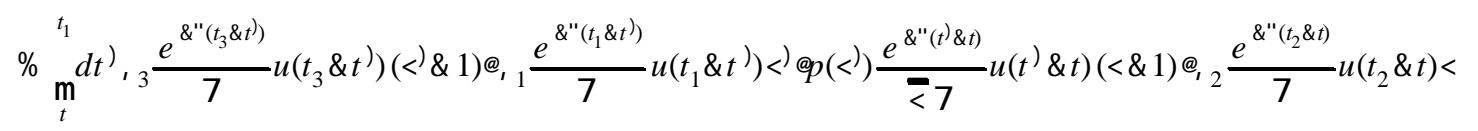

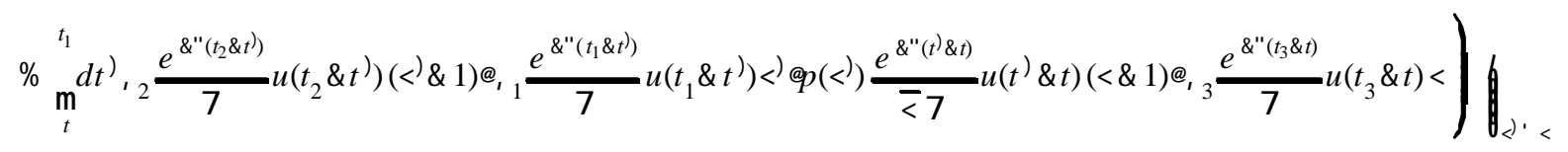

$$
\begin{aligned}
& (\hookrightarrow) \frac{e^{\Phi^{\prime \prime}\left(t \delta t_{0}\right)}}{\overline{<}} u\left(t \& t_{0}\right)<\varrho_{0}\left(<_{0}\right),{ }_{0} Q_{0}
\end{aligned}
$$

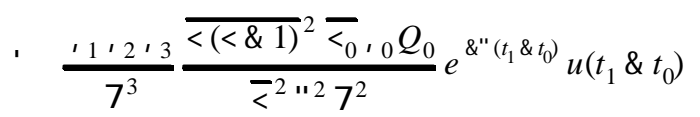

$$
\begin{aligned}
& \oint\left(e^{\varepsilon^{\prime \prime}\left(t_{2} \& t_{1}\right)} u\left(t_{2} \& t_{1}\right) \& e^{\delta^{\prime \prime}\left(t_{2} \& t_{0}\right)} u\left(t_{2} \& t_{0}\right)\right) e^{\varepsilon^{\prime \prime}\left(t_{3} \& t_{2}\right)} u\left(t_{3} \& t_{2}\right) \\
& \%\left(e^{\&^{\prime \prime}\left(t_{2} \& t_{1}\right)} u\left(t_{2} \& t_{1}\right) \& e^{\&^{\prime \prime}\left(t_{2} \& t_{0}\right)} u\left(t_{2} \& t_{0}\right)\right) e^{\&^{\prime \prime}\left(t_{3} \& t_{1}\right)} u\left(t_{3} \& t_{1}\right) \\
& \%\left(e^{\&^{\prime \prime}\left(t_{3} \& t_{1}\right)} u\left(t_{3} \& t_{1}\right) \& e^{\&^{\prime \prime}\left(t_{3} \& t_{0}\right)} u\left(t_{3} \& t_{0}\right)\right) e^{\& \alpha^{\prime \prime}\left(t_{2} \& t_{1}\right)} u\left(t_{2} \& t_{1}\right) \\
& \left.\& \frac{3}{2}\left(e^{\&^{\prime \prime}\left(t_{2} \& t_{1}\right)} e^{\varepsilon^{\prime \prime}\left(t_{3} \& t_{1}\right)} u\left(t_{2} \& t_{1}\right) u\left(t_{3} \& t_{1}\right) \& e^{\delta^{\prime \prime}\left(t_{2} \& t_{0}\right)} e^{\&^{\prime \prime}\left(t_{3} \varangle_{0}\right)} u\left(t_{2} \& t_{0}\right) u\left(t_{3} \& t_{0}\right)\right)\right) .
\end{aligned}
$$

The other five permutations in the sequence of detector counts simply correspond to permutations in the delays $\mathrm{J}_{1}{ }^{\prime} t_{1} \& t_{0}, \mathrm{~J}_{2}{ }^{\prime} t_{2} \& t_{0}$, and $\mathrm{J}_{3}{ }^{\prime} t_{3} \& t_{0}$ between each count and the initial source emission:

$$
\begin{aligned}
& \left(x_{0}, x_{1}, x_{2}, x_{3}\right): \mathrm{J}_{3}>\mathrm{J}_{2}>\mathrm{J}_{1}>0, \\
& \left(x_{0}, x_{1}, x_{3}, x_{2}\right): \mathrm{J}_{2}>\mathrm{J}_{3}>\mathrm{J}_{1}>0, \\
& \left(x_{0}, x_{2}, x_{1}, x_{3}\right): \mathrm{J}_{3}>\mathrm{J}_{1}>\mathrm{J}_{2}>0, \\
& \left(x_{0}, x_{2}, x_{3}, x_{1}\right): \mathrm{J}_{1}>\mathrm{J}_{3}>\mathrm{J}_{2}>0, \\
& \left(x_{0}, x_{3}, x_{1}, x_{2}\right): \mathrm{J}_{2}>\mathrm{J}_{1}>\mathrm{J}_{3}>0, \\
& \left(x_{0}, x_{3}, x_{2}, x_{1}\right): \mathrm{J}_{1}>\mathrm{J}_{2}>\mathrm{J}_{3}>0,
\end{aligned}
$$

such that the tricovariance $R_{0123}$ between the source and detector signals obeys the permutation relations

$$
\begin{aligned}
R_{0123}\left(\mathrm{~J}_{1}, \mathrm{~J}_{2}, \mathrm{~J}_{3}\right) & R_{0132}\left(\mathrm{~J}_{1}, \mathrm{~J}_{3}, \mathrm{~J}_{2}\right) \\
& R_{0213}\left(\mathrm{~J}_{2}, \mathrm{~J}_{1}, \mathrm{~J}_{3}\right) \\
\cdot & R_{0231}\left(\mathrm{~J}_{2}, \mathrm{~J}_{3}, \mathrm{~J}_{1}\right) \\
& R_{0312}\left(\mathrm{~J}_{3}, \mathrm{~J}_{1}, \mathrm{~J}_{2}\right) \\
& R_{0321}\left(\mathrm{~J}_{3}, \mathrm{~J}_{2}, \mathrm{~J}_{1}\right) .
\end{aligned}
$$


Consequently the tricovariance

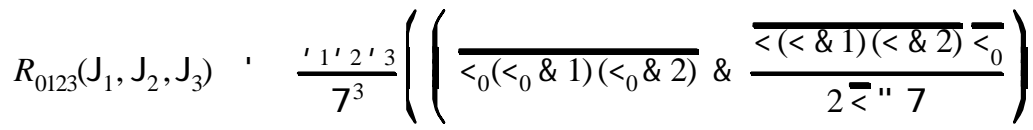

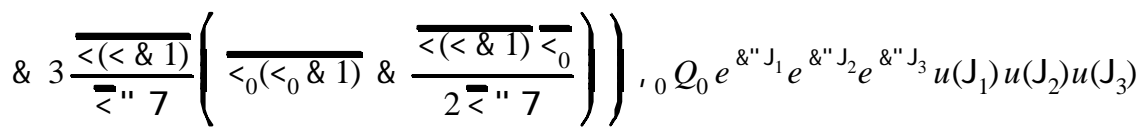

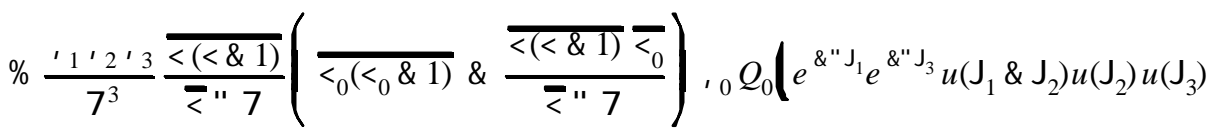

$$
\begin{aligned}
& \% e^{\& " J_{1}} e^{\& " J_{2}} u\left(J_{1} \& J_{3}\right) u\left(J_{2}\right) u\left(J_{3}\right) \% e^{\& \prime J_{1}} e^{\& " J_{2}} u\left(J_{1}\right) u\left(J_{2} \& J_{3}\right) u\left(J_{3}\right) \\
& \% e^{\& " J_{2}} e^{\& " J_{3}} u\left(J_{1}\right) u\left(J_{2} \& J_{1}\right) u\left(J_{3}\right) \% e^{\& " J_{2}} e^{\& " J_{3}} u\left(J_{1}\right) u\left(J_{2}\right) u\left(J_{3} \& J_{1}\right) \\
& \left.\% e^{\varepsilon^{\prime \prime} J_{1}} e^{\varepsilon^{\prime \prime} J_{3}} u\left(J_{1}\right) u\left(J_{2}\right) u\left(J_{3} \& J_{2}\right)\right)
\end{aligned}
$$

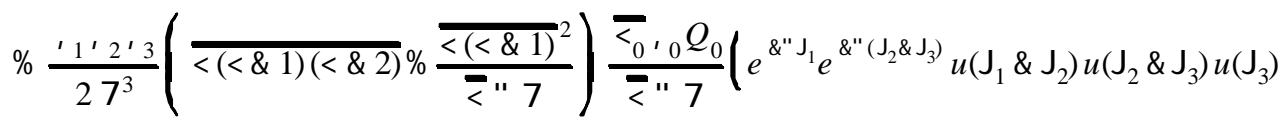

$$
\begin{aligned}
& \left.\% e^{\varepsilon^{\prime \prime} J_{1}} e^{\& \&^{\prime \prime}\left(J_{3} \& J_{2}\right)} u\left(J_{1} \& J_{2}\right) u\left(J_{2}\right) u\left(J_{3} \& J_{2}\right) \% e^{\& \prime J_{2}} e^{\left.\& J_{3} \& J_{1}\right)} u\left(J_{1}\right) u\left(J_{2} \& J_{1}\right) u\left(J_{3} \& J_{1}\right)\right)
\end{aligned}
$$

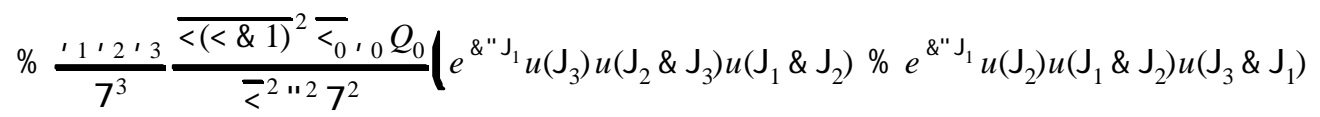

$$
\begin{aligned}
& \% e^{\& " J_{2}} u\left(J_{3}\right) u\left(J_{1} \& J_{3}\right) u\left(J_{2} \& J_{1}\right) \% e^{\& " J_{2}} u\left(J_{1}\right) u\left(J_{3} \& J_{1}\right) u\left(J_{2} \& J_{3}\right) \\
& \% e^{\& \prime J_{3}} u\left(J_{2}\right) u\left(J_{1} \& J_{2}\right) u\left(J_{3} \& J_{1}\right) \% e^{\& \prime J_{3}} u\left(J_{1}\right) u\left(J_{2} \& J_{1}\right) u\left(J_{3} \& J_{2}\right) \text { ) }
\end{aligned}
$$

is the distribution of correlated triplets of counts collectively chain-related to some detected active source emission over the delays $J_{1}, J_{2}$, and $J_{3}$ between each individual count in the triplet and the initial source emission. The corresponding trispectrum between the source and detector signals is the three-dimensional Laplace transform of the tricovariance:

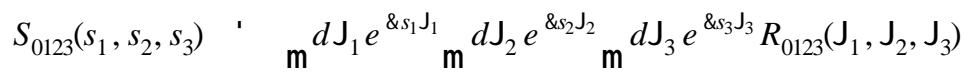

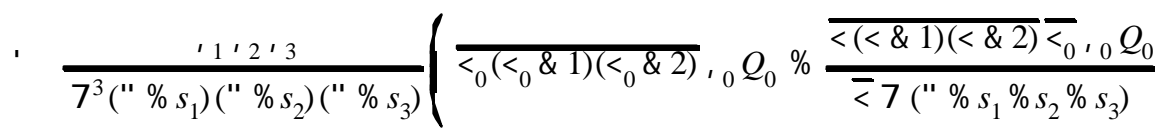

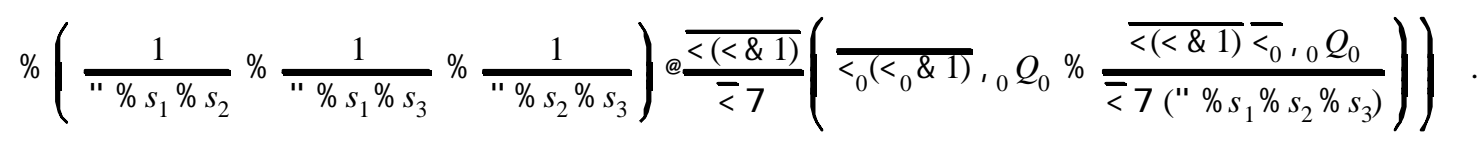

Observe that the trispectrum clearly distinguishes between the four processes that produce source-correlated triplets.

Next consider that correlated triplets can also arise from chain-reactions initiated by the unobservable sources " 0 " " and "G" in addition to the source " 0 " such that

$$
R_{123}\left(\mathrm{~J}_{1}, \mathrm{~J}_{2}\right) \quad R_{123}^{0}\left(\mathrm{~J}_{1}, \mathrm{~J}_{2}\right) \% R_{123}^{0(\mathrm{~J}}\left(\mathrm{J}_{1}, \mathrm{~J}_{2}\right) \% R_{123}^{\mathrm{G}}\left(\mathrm{J}_{1}, \mathrm{~J}_{2}\right)
$$


where the distribution of triplets chain-related to detected active source emissions can be obtained from the tricovariance according to

$$
\begin{aligned}
& R_{123}^{0}\left(\mathrm{~J}_{1}, \mathrm{~J}_{2}\right) \quad ' \quad \mathrm{rd}^{d \mathrm{~J}_{1}^{\prime}} R_{0123}\left(\mathrm{~J}_{1}^{\prime}, \mathrm{J}_{1}^{\prime} \% \mathrm{~J}_{1}, \mathrm{~J}_{1}^{\prime} \% \mathrm{~J}_{2}\right) \\
& \mathrm{m}^{d \mathrm{~J}_{2}^{\prime}} R_{0123}\left(\mathrm{~J}_{2}^{\prime} \& \mathrm{~J}_{1}, \mathrm{~J}_{2}^{\prime}, \mathrm{J}_{2}^{\prime} \% \mathrm{~J}_{2} \& \mathrm{~J}_{1}\right) \quad \mathrm{m}^{d \mathrm{~J}_{3}^{\prime}} R_{0123}\left(\mathrm{~J}_{3}^{\prime} \& \mathrm{~J}_{2}, \mathrm{~J}_{3}^{\prime} \& \mathrm{~J}_{2} \%_{1}, \mathrm{~J}_{3}^{\prime}\right)
\end{aligned}
$$

The distribution of triplets chain-related to the two other sources can be obtained by simply substituting them for the source " 0 " in (50) through (53) and (59). Consequently, the bicovariance between detector signals

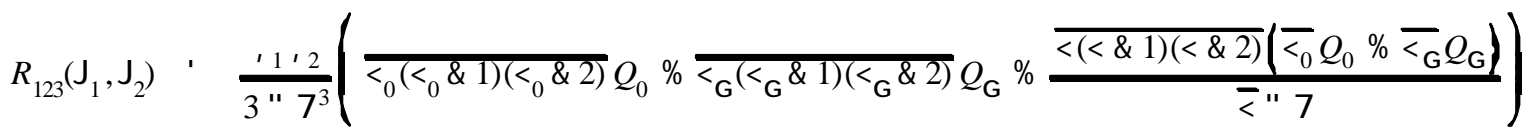

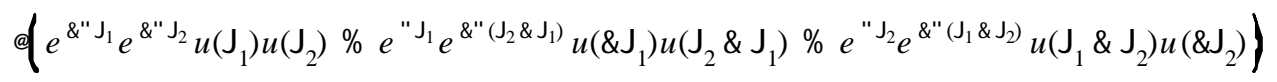

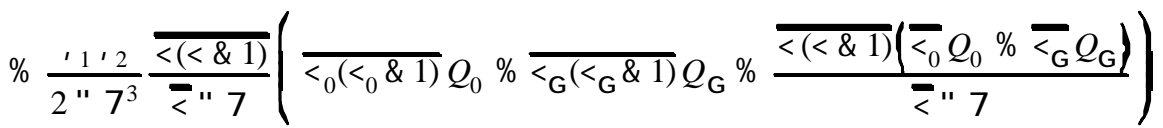

$$
\begin{aligned}
& \oint e^{\& \prime J_{2}} u\left(J_{1}\right) u\left(J_{2} \& J_{1}\right) \% e^{\& \prime J_{1}} u\left(J_{1} \& J_{2}\right) u\left(J_{2}\right) \% e^{\left.\& J^{\prime \prime} J_{2} \& J_{1}\right)} u\left(\& J_{1}\right) u\left(J_{2}\right) \\
& \left.e^{" J_{1}} u\left(J_{2} \& J_{1}\right) u\left(\& J_{2}\right) \% e^{\& "\left(J_{1} \& J_{2}\right)} u\left(J_{1}\right) u\left(\& J_{2}\right) \% e^{n J_{2}} u\left(\& J_{1}\right) u\left(J_{1} \& J_{2}\right)\right)
\end{aligned}
$$

is the distribution of all correlated triplets of counts over the delay $\mathrm{J}_{1}$ between the first and second count and $\mathrm{J}_{2}$ between the first and third count (see also [7]). The bispectrum between detectors is the two-dimensional Laplace transform of the bicovariance; like the bicovariance, the bispectrum has components corresponding to each of the three sources " 0 ", " 0 ", and "G":

$$
S_{123}\left(s_{1}, s_{2}\right) \quad \mathrm{m}^{\left.d \mathrm{~J}_{1} e^{\delta_{1} \mathrm{~J}_{1}} \mathrm{~m}^{d \mathrm{~J}_{2}} e^{\delta_{2} \mathrm{~J}_{2}} R_{123} \mathrm{~J}_{1}, \mathrm{~J}_{2}\right) \quad ' \quad S_{123}^{0}\left(s_{1}, s_{2}\right) \% S_{123}^{0^{d}}\left(s_{1}, s_{2}\right) \% S_{123}^{\mathrm{G}}\left(s_{1}, s_{2}\right)} .
$$

In accordance with (59), the bispectrum component corresponding to detected active source emissions

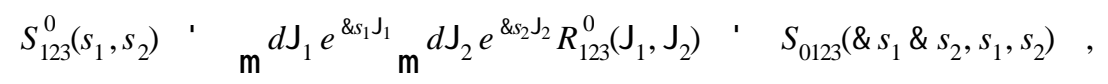

and the other two components can be obtained by substitution such that

$$
\begin{aligned}
& S_{123}\left(s_{1}, s_{2}\right) \quad \text { ' } \frac{1_{1}, 2,3}{7^{3}\left(" \& s_{1} \& s_{2}\right)\left(" \% s_{1}\right)\left(" \% s_{2}\right)}
\end{aligned}
$$

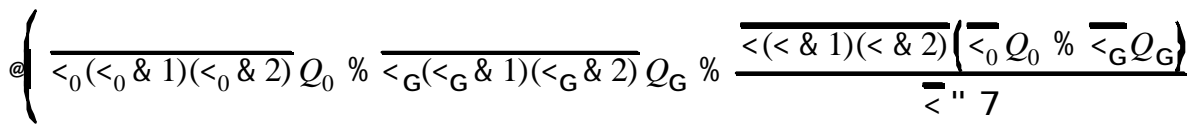

$$
\begin{aligned}
& \%\left(\frac{1}{" \& s_{1}} \% \frac{1}{" \varepsilon s_{2}} \% \frac{1}{" \% s_{1} \% s_{2}}\right)
\end{aligned}
$$

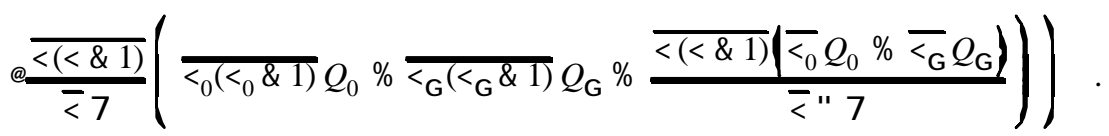

Next consider that one can define a source-conditional bicovariance $R_{123 *_{0}}$ between detectors 


$$
R_{0123}\left(\mathrm{~J}_{1}, \mathrm{~J}_{2}, \mathrm{~J}_{3}\right) \quad ' \quad R_{123} *_{0}\left(\mathrm{~J}_{1}, \mathrm{~J}_{2}, \mathrm{~J}_{3}\right) @_{0}
$$

and a corresponding conditional mean $\overline{\left(x_{1} x_{2} x_{3}\right)_{* 0}}$ such that

$$
\begin{aligned}
& \frac{\overline{\left(x_{1} x_{2} x_{3}\right)_{*}}}{\overline{\left(x_{1} x_{2} x_{3}\right)} / \overline{x_{0}}} \cdot \frac{\mathrm{m}^{d \mathrm{~J}_{1}} \mathrm{~m}^{d \mathrm{~J}_{2}} \mathrm{~m}^{d \mathrm{~J}_{3} R_{123} *_{0}\left(\mathrm{~J}_{1}, \mathrm{~J}_{2}, \mathrm{~J}_{3}\right)}}{\frac{1}{\overline{x_{0}}} \mathrm{~m}^{d \mathrm{~J}_{1}} \mathrm{~m}^{d \mathrm{~J}_{2} R_{123}\left(\mathrm{~J}_{1}, \mathrm{~J}_{2}\right)}} \cdot \frac{\mathrm{m}^{d \mathrm{~J}_{1}} \mathrm{~m}^{d \mathrm{~J}_{2}} \mathrm{~m}^{d \mathrm{~J}_{3} R_{0123}\left(\mathrm{~J}_{1}, \mathrm{~J}_{2}, \mathrm{~J}_{3}\right)}}{\mathrm{m}^{d \mathrm{~J}_{1}} \mathrm{~m}^{d \mathrm{~J}_{2} R_{123}\left(\mathrm{~J}_{1}, \mathrm{~J}_{2}\right)}} \cdot \frac{S_{0123}(0,0,0)}{S_{123}(0,0)}
\end{aligned}
$$

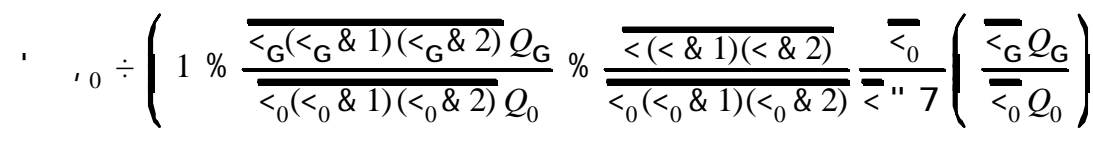

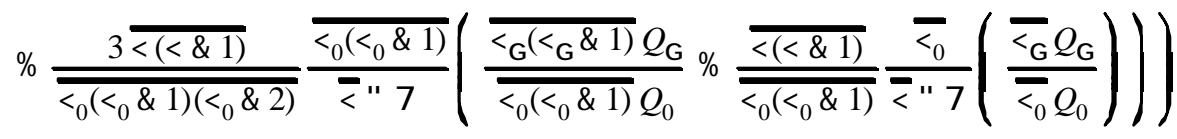

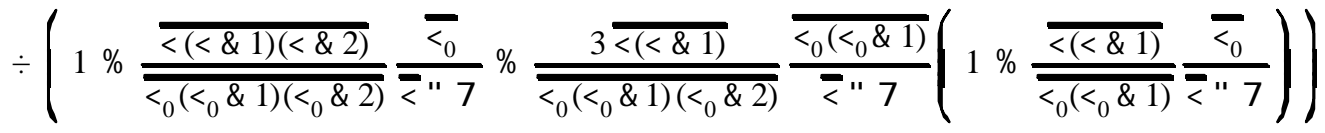

is the fraction of all correlated triplets of counts that are collectively chain-related to a detected source emission. Hence it possible to distinguish triplets chain-related to the active source from those chain-related to other sources.

\subsection{GENERALIZATION TO ARBITRARY ORDER}

For obvious reasons, it is desirable to develop a simple "prescription" to predict statistics of arbitrary order $n$. Because, for a given order $n$, the mutually exclusive processes that lead to $n$ correlated counts can be represented diagrammatically, the first step in this development will be a procedure to ensure that all diagrams representing a particular order are generated.

If the individual symbols in each diagram are thought of as "words," then there are some simple recursive rules of composition to form all "sentences" that represent a given order. This is illustrated in Fig. 6 for orders one through five. ${ }^{11}$ First observe that the notation for each diagram is equivalent to that diagram's order (e.g., " $(2-2)+2+3$ " = order 5). Next consider that the diagrams along the leftmost column are "minimal" symbols representing each order. Each of these symbols has $n$ branches and so is defined to be equivalent to the number $n$. They are called "minimal" because, for each order, the minimal symbols are the only diagrams composed of one symbol representing that order. Subsequent to these are composite (i.e., non-minimal) diagrams formed by concatenation of minimal symbols. Note that composite diagrams of order $n$ contain only minimal symbols with equivalent values less than or equal to $n$.

Observe that each right-concatenated symbol is defined to be equivalent to its number of branches, while each leftconcatenated symbol is defined to be equivalent to its number of branches minus the number of symbols right-concatenated to it. Consider all the "two-stage" composite diagrams (e.g., "(2-2)+2+3") formed by right-concatenating $m$ minimal symbols to a single minimal symbol on the left with $n \&$ Rbranches. The basic rule of composition for these diagrams is

$$
\begin{gathered}
\left.n^{\prime}(\mid n \& \mathrm{R}] \& m\right\} \% \mathrm{E}_{m}, \\
m 0\left\{1,{ }^{\vee},\lfloor n / 2\rfloor\right\}, \quad \operatorname{RO}\left\{m,{ }^{\nu}, n \& m\right\},
\end{gathered}
$$

where [ $n \& \mathrm{R}]$ denotes the equivalent value of the symbol with $n \&$ Rbranches, and "I l" denotes the "floor" operator that yields the integer less than or equal to its operand. For example, for the composite "(2-2)+2+3", $n^{\prime} 5, m^{\prime}$, and $\mathrm{R}^{\prime} 3$. $\mathrm{E}_{m}$

${ }^{11}$ The diagrams beginning with "(1-1)" are not shown in Fig. 6 because they are simply composed of the symbol for
"1" left-concatenated to the diagrams already shown. 
is a sum with $m$ terms, each corresponding to the equivalent value of one right-concatenated symbol, such that $\mathrm{E}_{m}$ sums to $m \% \mathbf{R}$

$$
\begin{gathered}
\mathrm{E}_{m}{ }^{\prime} k_{1} \% k_{2} \%, \% k_{m} * \mathrm{E}_{m}{ }^{\prime} m \% \mathrm{R}, \\
k_{m} \$ k_{m \& 1} \$ 。 \$ k_{1} \$ 2 .
\end{gathered}
$$

For example, for the composite "(2-2)+2+3", $k_{1}{ }^{\prime} 2$ and $k_{2}{ }^{\prime} 3$. For Rand $m$ restricted to the ranges listed in (66), it will always be possible to construct $\mathrm{E}_{m}$ from $m$ terms each greater than or equal to two because

$$
2 m \quad \# \quad \mathrm{E}_{m} \quad \# \quad n
$$

Finally, each term of $\mathrm{E}_{m}$ with an equivalent value greater than two can be recursively expanded in the composite diagrams for the order equal to its equivalent value. For example, because " $(2-1)+2$ " is the composite diagram for order 3 , " $(2-2)+2+3$ " can be expanded into " $(2-2)+2+(2-1)+2$ ". This recursive rule includes composite diagrams of the form " $(1-1)+n$ ". The single term, $n$, of $\mathrm{E}_{m^{\prime} 1}$ can be expanded into all the preceding composite diagrams representing order $n$. In this manner all composite diagrams with more than two stages can be recursively generated. This recursive feature of the composition rules results in the complete and non-redundant generation of all diagrams of a given order. Table 1 lists the resulting expansions for all diagrams of order one through six. Note in particular that successive application of the recursion rule always terminates in a composite of the form " $(1-1)+(2-1)+(2-1)+\ldots+(2-1)+2$ ". This "maximal” word represents the longest composite diagram representing a given order. Later, both the minimal and maximal diagrams will be used to prescribe bounds on the behavior of statistics of arbitrary order.

For a given order $n$, each diagram is equivalent to the joint probability for a particular sequence of events terminating in an $n$-tuplet of detector counts. Because all the diagrams for a particular order represent mutually exclusive sequences of events, the total joint probability for that order is simply the sum of the joint probabilities represented by the diagrams. Furthermore, due to the equivalence between the $n$ th-order poly-covariance and the joint probability for that order, the diagrams are equivalent to the $n$ th-order poly-covariance. Finally, because there is an isomorphism between the $n$ th-order poly-spectrum and poly-covariance, the diagrams are equivalent to the $n$ th-order poly-spectrum. The rules for expressing a particular diagram as a poly-spectrum are fairly simple.

First consider the $n$ th-order poly-spectrum between an active source signal and $n$ detector signals. Each event in a particular permutation of a sequence contributes a factor to the poly-spectrum. The $i$ th terminal (detection) event contributes a factor of

$$
\frac{{ }^{i}}{7\left(" \% s_{i}\right)}, \quad i 0\{1, \stackrel{v}{,}, n\}
$$

Each intermediate (induced fission) event contributes a factor of

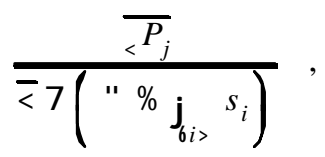

where $(i$ : denotes the set of terminal events traceable through the diagram to that particular intermediate event. The $j$ th factorial moment of $p(\triangleleft)$ is denoted by

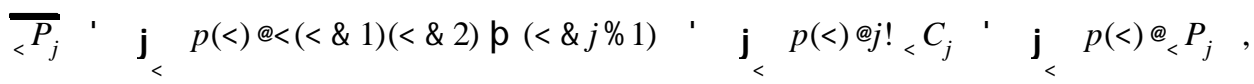

where $j$ is the number of branches in the symbol representing the induced fission event. Note also that all possible permutations of the subset $\left(i:\right.$ of $\left(1,2,{ }^{\vee}, n\right.$ : must be included as additive terms and that multiple intermediate events in the 
same stage trace to mutually exclusive sets of terminal events (i.e., multiple fissions events cannot cause the same detection event). Finally, the initial (source emission) event contributes a factor of

$$
{ }_{{ }_{8}} P_{k},{ }_{0} Q_{0}
$$

where $k$ is the number of branches in the initial symbol in the diagram. For example, the fourth-order diagram " $(2-1)+(2-1)+2$ " is equivalent to the term

$$
\begin{aligned}
& (2 \& 1) \% 2 \& 1) \% S_{01234}\left(s_{1}, s_{2}, s_{3}, s_{4}\right) \quad \text { ' } \frac{\prime^{\prime} 2^{\prime} 3^{\prime} 4}{7^{4}\left(" \% s_{1}\right)\left(" \% s_{2}\right)\left(" \% s_{3}\right)\left(" \% s_{4}\right)} \\
& \text { @ } \overline{\overline{<}(<\& 1)}\left(\frac{1}{" \% s_{1} \% s_{2}} \% \frac{1}{" \% s_{1} \% s_{3}} \% \frac{1}{" \% s_{1} \% s_{4}} \% \frac{1}{" \% s_{2} \% s_{3}} \% \frac{1}{" \% s_{2} \% s_{4}} \% \frac{1}{" \% s_{3} \% s_{4}}\right) \\
& \text { @ } \frac{\overline{<<1)}}{\overline{<} 7}\left(\frac{1}{" \% s_{1} \% s_{2} \% s_{3}} \% \frac{1}{" \% s_{1} \% s_{2} \% s_{4}} \% \frac{1}{" \% s_{1} \% s_{3} \% s_{4}} \% \frac{1}{" \% s_{2} \% s_{3} \% s_{4}}\right) \\
& \overline{\varnothing_{0}(<\& 1)},{ }_{0} Q_{0}
\end{aligned}
$$

in the fourth-order poly-spectrum between the active source and detector signals, and the diagram " $(2-2)+2+2$ " is equivalent to the term

$$
\begin{aligned}
& { }_{(2 \& 2) \% \%} S_{01234}\left(s_{1}, s_{2}, s_{3}, s_{4}\right) \quad \frac{\prime^{\prime} 2^{\prime} 3{ }^{\prime} 4}{7^{4}\left(" \% s_{1}\right)\left(" \% s_{2}\right)\left(" \% s_{3}\right)\left(" \% s_{4}\right)}
\end{aligned}
$$

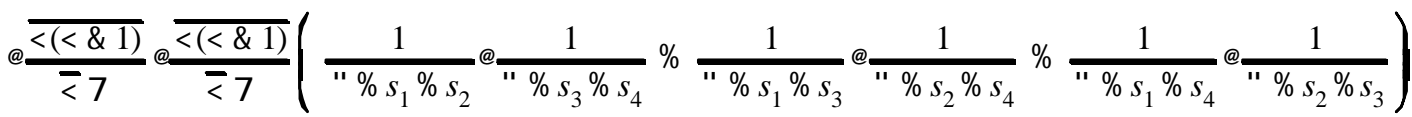

$$
\begin{aligned}
& \overline{\mathbb{Q}_{0}(\zeta \& 1)},{ }_{0} Q_{0}
\end{aligned}
$$

in this same poly-spectrum.

Once the $n$ th-order poly-spectrum between the active source and detector signals has been determined, it is easy to generate the $n$ th-order poly-spectrum between only the detector signals. This is done via two substitutions. First,

$$
\overline{{ }_{\delta} P_{k},{ }_{0}} Q_{0} \quad 6 \overline{{ }_{\delta} P_{k}} Q_{0} \% \overline{{ }_{\xi} P_{k}} Q_{\mathrm{G}}
$$

because all active and intrinsic source emissions can potentially produce a correlated $n$-tuplet of detector counts. Second,

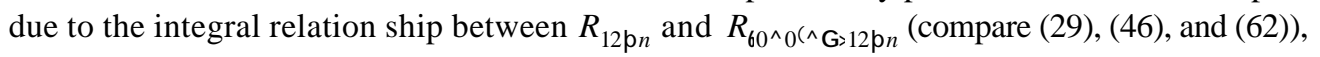

$$
\begin{array}{rlllll}
s_{1} \% s_{2} \%, \% s_{n} & 6 \quad 0 \quad & s_{1} & 6 & \& s_{1} \& s_{2} \&, \& s_{n \& 1} \\
& s_{2} & 6 & s_{1} \\
s_{3} & 6 & s_{2} \\
& ! & \\
s_{n} & 6 & s_{n \& 1} .
\end{array}
$$

For example, the fourth-order diagram “ $(2-1)+(2-1)+2$ ” is equivalent to the term 


$$
\begin{aligned}
& { }_{(2 \& 1) \%(2 \& 1) \%} S_{1234}\left(s_{1}, s_{2}, s_{3}\right) \quad ' \frac{,_{1}, 2,3,4}{7^{4}\left(" \& s_{1} \& s_{2} \& s_{3}\right)\left(" \% s_{1}\right)\left(" \% s_{2}\right)\left(" \% s_{3}\right)} \\
& \text { @ } \frac{\overline{<<\& 1)}}{\bar{\gtrless}}\left(\frac{1}{" \& s_{2} \& s_{3}} \% \frac{1}{" \& s_{1} \& s_{3}} \% \frac{1}{" \& s_{1} \& s_{2}} \% \frac{1}{" \% s_{1} \% s_{2}} \% \frac{1}{" \% s_{1} \% s_{3}} \% \frac{1}{" \% s_{2} \% s_{3}}\right)
\end{aligned}
$$

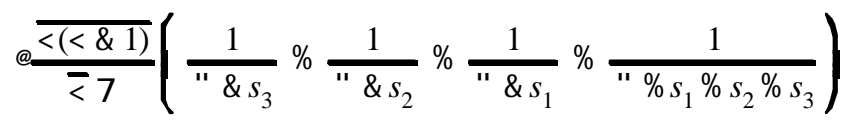

$$
\begin{aligned}
& \text { фर्大(<\&1) } \left.Q_{0} \% \overline{<_{G}\left(<_{G} \& 1\right)} Q_{G}\right)
\end{aligned}
$$

in the fourth-order poly-spectrum between detector signals, and the diagram " $(2-2)+2+2$ " is equivalent to the term

$$
\begin{aligned}
& { }_{(2 \&) \% \% \%} S_{1234}\left(s_{1}, s_{2}, s_{3}\right) \quad ' \frac{\rho_{1}, 2,3,4}{7^{4}\left(" \& s_{1} \& s_{2} \& s_{3}\right)\left(" \% s_{1}\right)\left(" \% s_{2}\right)\left(" \% s_{3}\right)}
\end{aligned}
$$

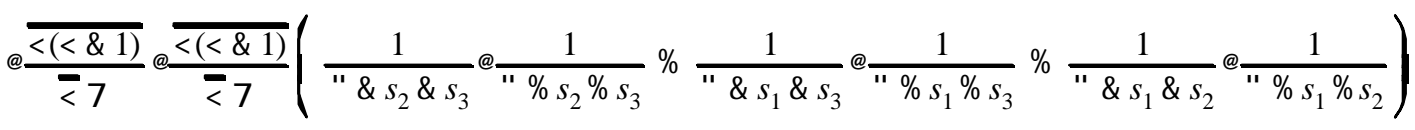

$$
\begin{aligned}
& \text { ф<(<\&1)} \left.Q_{0} \% \overline{<_{G}\left(<_{G} \& 1\right)} Q_{G}\right)
\end{aligned}
$$

in this same poly-spectrum.

So the preceding is the prescription required to predict poly-spectra of arbitrary order. The corresponding polycovariances can be synthesized via application of the inverse-Laplace transform. Unfortunately, a prescription to predict $n$ thorder poly-covariances, although implicit in the preceding procedure, has not yet been explicitly formulated. ${ }^{12}$ However, it is not absolutely necessary to possess such a procedure. The subsequent general features of higher order statistics can be fairly easily described in terms of poly-spectra.

\section{GENERAL PROPERTIES OF HIGH ORDER STATISTICS AND THEIR POTENTIAL UTILITY}

Frequently it is easier to describe the properties of something new in terms of the new things that can be done with it. Furthermore, because "newness" is only a state relative to preceding developments, the following contains several comparisons of applications of the new statistics to similar applications using previously developed ones. To date, two potentially useful general properties of higher order statistics have been identified. First, they can be used to distinguish between the temporal distribution of $n$-tuplets that are chain-related to an introduced active source from that of $n$-tuplets that are chain-related to an intrinsic source that is otherwise unobservable. Second, successively higher order statistics possess progressively higher sensitivity to changes in reactivity and hence to changes in composition, configuration, and fissile mass.

On several occasions it has been noted that a poly-covariance between an active source and $n$ detectors can be used in conjunction with a poly-covariance between $n$ detectors to determine the fraction of all $n$-tuplets that are correlated to a detected active source emission (see (32), (48), and (65)). This is because the poly-covariance

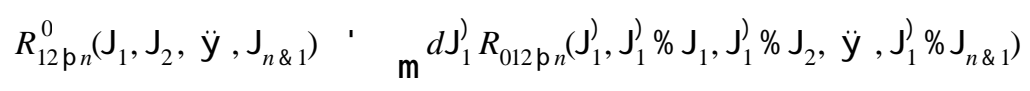

\footnotetext{
${ }^{12}$ Hopefully, this desired formulation will be evident to some interested reader.
} 
is the temporal distribution of $n$-tuplets that are correlated to a detected active source emission while the poly-covariance $R_{12, n}$ is the temporal distribution of all $n$-tuplets. In principle, this relationship could be used to distinguish between the distribution of $n$-tuplets chain-related to the active source from that of $n$-tuplets chain-related to the intrinsic source, if one is present, because

$$
R_{12, n}\left(\mathrm{~J}_{1}, \mathrm{~J}_{2}, \vee, \mathrm{J}_{n \& 1}\right) \quad ' \quad R_{12, n}^{0}\left(\mathrm{~J}_{1}, \mathrm{~J}_{2}, \stackrel{\vee}{ }, \mathrm{J}_{n \& 1}\right) \% R_{12, n}^{\tilde{0}}\left(\mathrm{~J}_{1}, \mathrm{~J}_{2}, \vee, \mathrm{J}_{n \& 1}\right)
$$

In other words, the marginal distribution $R_{12, n}$ is comprised of one component $R_{12, n}^{0}$ that is due to detected active source emissions and one component $R_{12, n}^{\tilde{0}}$ that is not. One problem in the implementation of this principle is that the active source may not be $100 \%$ efficient, and the efficiency of the active source may be unknown. That is

$$
R_{12, n}^{\tilde{0}}\left(\mathrm{~J}_{1}, \mathrm{~J}_{2}, \vee \mathrm{J}_{n \& 1}\right) \quad ' \quad R_{12,{ }_{n}}^{0^{(}}\left(\mathrm{J}_{1}, \mathrm{~J}_{2}, \stackrel{\vee}{ }, \mathrm{J}_{n \& 1}\right) \% R_{12,{ }_{n}}^{\mathrm{G}}\left(\mathrm{J}_{1}, \mathrm{~J}_{2},{ }^{\vee}, \mathrm{J}_{n \& 1}\right)
$$

where $R_{12, n}^{0^{r}}$ is due to undetected active source emissions and $R_{12, n}^{\mathrm{G}}$ is due to the intrinsic source.

However, a solution to this problem lies within the principle itself. If the poly-covariance between the active source and detectors and the poly-covariance between just the detectors are measured simultaneously in the absence of any fissile system or intrinsic source, then

$$
\begin{aligned}
& p(<>1) 60, \quad Q_{\mathrm{G}} 60:
\end{aligned}
$$

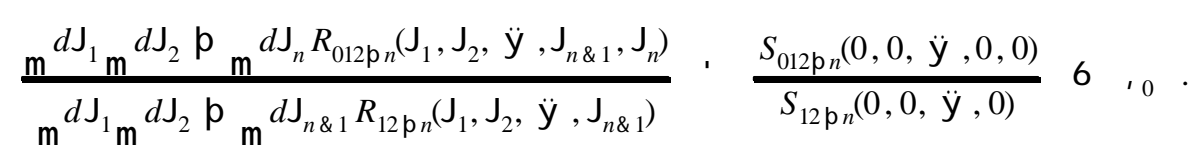

For example, the efficiency of a ${ }^{252} \mathrm{Cf}$ ionization chamber can be measured by using two detectors and acquiring the bicovariance between the source and detectors and the covariance between the detectors when the two detectors are exposed to the source in air in the absence of any other spontaneous fission sources. ${ }^{13}$ The author and colleagues at the Oak Ridge National Laboratory have performed such measurements using plastic scintillators and constant fraction discrimination pulse processing instrumentation to optimize the discriminator threshold for ${ }^{252} \mathrm{Cf}$ ionization chambers and achieve maximum efficiency. ${ }^{14}$ During these measurements it was noted that the efficiency measured for any particular source threshold was completely insensitive to changes in the detector thresholds, the high voltage applied to the detector photomultiplier tubes, the coincidence gate width used to collect the covariance and bicovariance, and the physical location of the detectors and their proximity to the ${ }^{252} \mathrm{Cf}$ source. So, for this particular method, the conclusion (82) drawn from the supporting theory extends beyond the limitations of the point models used to develop the theory.

Once the source efficiency has been measured, subsequent measurements of a fissile system containing intrinsic sources can partition the distribution of $n$-tuplets $R_{12, n}$ into one component $R_{12,{ }_{n}}^{0^{\wedge} 0^{\prime}}$ that is due to all active source emissions

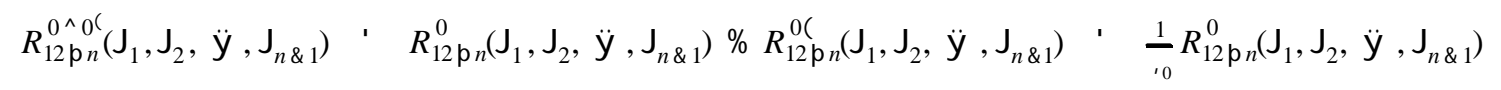

\footnotetext{
${ }^{13}$ Observe that only first-order statistics (i.e., the covariance between the source and a detector and the detector count rate) can be used to measure the efficiency of an active source that emits only one neutron per source event (e.g., an APNG) because $p_{0}(\zeta>1)^{\prime} 0$, and then the measurement must be performed in the absence of significant background.
}

${ }^{14}$ Sometimes it is not possible to select a threshold to achieve $100 \%$ counting efficiency for ${ }^{252} \mathrm{Cf}$ fission-fragments because it is necessary to ensure that all counts due to $\alpha$-decay of ${ }^{252} \mathrm{Cf}$ are discriminated. This often discriminates some low energy fission-fragment counts as well.[1] 
and one component $R_{12, n}^{\mathrm{G}}$ that is due only to intrinsic source emissions such that

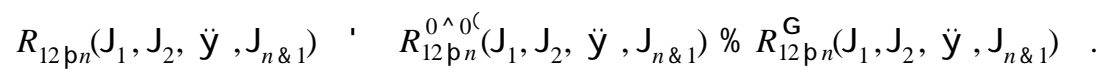

Consequently, the distribution of $n$-tuplets chain-related to intrinsic source emissions can be indirectly measured. This means that active measurements performed on fissile systems containing intrinsic sources, e.g., plutonium assemblies or spent nuclear fuel, can now use this technique to measure properties of the otherwise unobservable intrinsic sources.

Sometimes, however, the properties of intrinsic sources are of no real interest. In such applications, it is desirable to measure quantities that are independent of the presence and/or strength of intrinsic sources. Furthermore, it is also desirable to construct quantities that are as well independent of detection efficiency and the response of associated counting instrumentation.

Discussion of the effects of instrumentation response has been deferred up to this point. Now consider that the signal $x_{m}^{\prime}\left(t_{m}^{\prime}\right)$ that is actually acquired from channel $m$ (which can be either an active source or a radiation detector) is related to the actual sequence of counting events $x_{m}\left(t_{m}\right)$ by

$$
x_{m}^{\prime}\left(t_{m}^{\prime}\right) \quad \mathrm{n}^{d t_{m}} h_{m}\left(t_{m}\right) x_{m}\left(t_{m}^{\prime} \& t_{m}\right)
$$

where $h_{m}$ is the impulse response of the electronics associated with channel $m$. Subsequently, the transfer function of the electronics associated with channel $m$ is

$$
H_{m}(s) \quad \mathrm{n}^{d t e^{\delta s t} h_{m}(t)}
$$

Upon inclusion of the electronics transfer functions, the acquired poly-spectrum between the source and detectors becomes

$$
S_{012, n}\left(s_{1}, s_{2}, \stackrel{v}{,}, s_{n}\right) \quad 6 H_{0}\left(\& s_{1} \& s_{2} \&, \& s_{n}\right) H_{1}\left(s_{1}\right) H_{2}\left(s_{2}\right), H_{n}\left(s_{n}\right) \bigotimes_{012, n}\left(s_{1}, s_{2}, \quad, \quad, s_{n}\right),
$$

and the acquired poly-spectrum between detectors becomes ${ }^{15}$

$$
S_{12, n}\left(s_{1}, s_{2}, `, s_{n \& 1}\right) \quad 6 H_{1}\left(\& s_{1} \& s_{2} \&, \& s_{n \& 1}\right) H_{2}\left(s_{1}\right) H_{3}\left(s_{2}\right), H_{n}\left(s_{n \& 1}\right) \bigotimes_{12, n}\left(s_{1}, s_{2}, \stackrel{v}{,}, s_{n \& 1}\right) .
$$

Consequently, if the impulse response of the electronics associated with any channel changes, as can occur due to changes in gain or timing resolution, these statistics will change accordingly.

In order to construct a quantity that would be robust to changes in detection efficiency and electronics response, Mihalczo and Paré introduced the spectral ratio ${ }^{16}[10]$

$$
{ }_{012}(s) \quad, \quad \frac{S_{01}(\& s) S_{02}(s)}{S_{00}(\& s) S_{12}(s)},
$$

where $S_{00}$ is the source autospectrum (a univariate statistic)

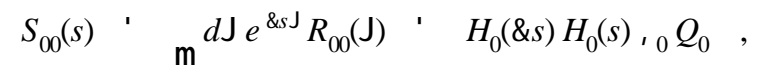

${ }^{15}$ Observe that upon inclusion of the source and detector transfer functions, (82) yields ${ }_{0}{ }_{0} H_{0}(0)$, where $H_{0}(0){ }^{\prime}{ }^{*} d t h_{0}(t)$ is essentially the gain of the source electronics. However (82) still yields the fraction of all active source events that are actually counted.

${ }^{16}$ In earlier publications, this quantity has been referred to as the ratio of spectral densities; more recent publications refer to it as the spectral ratio.[10] 
where $R_{00}$ denotes the source autocovariance (another univariate statistic), that, upon inclusion of the source impulse response, is

$$
\begin{gathered}
R_{00}() \quad 6 \mathrm{rr}^{d t_{0} \mathrm{~m}} d t_{0}^{\prime} h_{0}\left(t_{0}\right) h_{0}\left(t_{0}^{\prime}\right) R_{00}\left(\% t_{0} \& t_{0}^{\prime}\right) \\
\mathrm{m}^{d t_{0}} \mathrm{~m}^{d t_{0}^{\prime}} h_{0}\left(t_{0}\right) h_{0}\left(t_{0}^{\prime}\right),{ }_{0} Q_{0} *\left(\mathrm{~J} \% t_{0} \& t_{0}^{\prime}\right) \quad \mathrm{m}^{d t_{0} h_{0}\left(t_{0}\right) h_{0}\left(t_{0} \% \mathrm{~J}\right),{ }_{0} Q_{0}}
\end{gathered}
$$

Observe that although the spectral ratio is independent of the detectors' efficiencies and the impulse response of any associated electronics, it does depend upon the source efficiency and upon the presence and strength of intrinsic sources

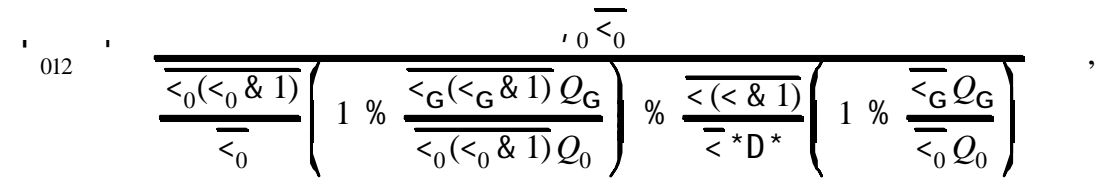

where $* D^{k^{\prime}} * \&^{\prime \prime} 7 *$ denotes subcritical prompt reactivity. Furthermore, although not explicitly shown, the inherent delayed neutron source will as well affect the spectral ratio according to

$$
\overline{<} Q \quad 6 \quad(1 \% \$)<Q \quad, \quad \frac{(1 \% \$)}{* D^{*}}\left(\bar{\gamma} Q_{0} \% \overline{<}_{\mathrm{G}} Q_{\mathrm{G}}\right),
$$

where \$ denotes the system's effective delayed neutron fraction.

Consider as a modification to the spectral ratio the quantity

$$
\mathrm{Q}_{1 @ * 0} \quad \frac{S_{01}(0) S_{02}(0)}{\overline{x_{0}} S_{012}(0,0)}
$$

that, for lack of a better name, will be called the bispectral ratio. Observe that

$$
\mathrm{Q}_{1 @ * 0} \cdot \frac{\left.\left(\mathrm{m} d \mathrm{~J} R_{1 *_{0}}(\mathrm{~J})\right) \Phi_{\mathrm{m}} d \mathrm{~J} R_{2 * 0}(\mathrm{~J})\right)}{\mathrm{m}^{d \mathrm{~J}^{1}} \mathrm{~m}^{d \mathrm{~J}_{2} R_{12 * 0}\left(\mathrm{~J}_{1}, \mathrm{~J}_{2}\right)}}
$$

such that the bispectral ratio can be thought of as the number of independent pairs whose individual members are chainrelated to the active source per correlated pair that is collectively chain-related to the active source. This quantity,

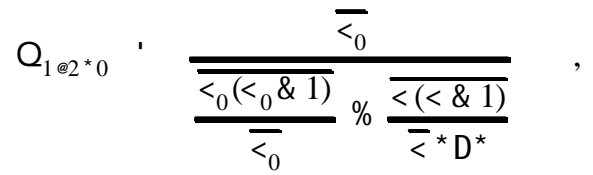

is independent of both the detectors' efficiencies and the source efficiency as well as the impulse response of any associated electronics. Furthermore, because all quantities in the bispectral ratio are correlated to the active source, it is completely independent of the presence and/or strength of any intrinsic sources. Finally, because the inherent delayed neutron source is essentially uncorrelated with active source emissions, the bispectral ratio is also independent of the strength of the inherent delayed neutron source.

Further observe that as a subcritical fissile system approaches prompt critical,

$$
\mathrm{Q}_{1 @ * 0} \operatorname{xaxv}_{\mathrm{D} 60} \frac{\bar{\delta}<* D^{*}}{\overline{<(<\& 1)}}
$$


i.e., the bispectral ratio is directly proportional to subcritical prompt reactivity for systems close to prompt critical. Next observe that for extremely subcritical systems, the bispectral ratio asymptotically approaches the source Diven factor:

$$
\mathrm{Q}_{1 @ * 0} \underset{\mathrm{D} 6 \& 4}{\operatorname{xxxV}} \frac{\bar{\zeta}^{2}}{\overline{\delta_{0}\left(\zeta_{0} \& 1\right)}}
$$

if an active source capable of emitting multiple neutrons per event is used. Monte Carlo calculational models of ${ }^{25} \mathrm{Cf}$ sourcedriven measurements of simple uranium-metal systems indicate that the transition between asymptotic behaviors (97) and (98) occur in the region where the prompt multiplication factor $k-0.5$.[20] Consequently, it may be possible to use the bispectral ratio to estimate subcritical prompt reactivity for $k / 0.5$.

If, on the other hand, an active source that emits only a single neutron per event (e.g., an APNG) is used, then the bispectral ratio is directly proportional to subcritical prompt reactivity

$$
p_{0}(\zeta>1), 0: \quad 0 \quad \mathrm{Q}_{1 @ * 0}, \frac{\bar{\zeta} \overline{<}^{*} \mathrm{D}^{*}}{\overline{<(<\& 1)}}
$$

for all values of subcritical reactivity. Consequently, it may be possible to use the bispectral ratio to estimate subcritical prompt reactivity over a broad range if an APNG is used as the active source. Furthermore, measurements of uranium-metal systems have shown that not only is the bispectral ratio independent of detection efficiency but that it is also fairly robust to changes in the position of the source and detectors relative to one another and to the fissile system. So, although only a few measurements of this quantity have as yet been conducted, it is reasonable to speculate that the bispectral ratio may be of significant utility for monitoring subcritical reactivity even in fissile systems containing unknown intrinsic sources, e.g., spent nuclear fuel and plutonium assemblies.

Observe in addition that it is also possible to construct higher order poly-spectral ratios of the form

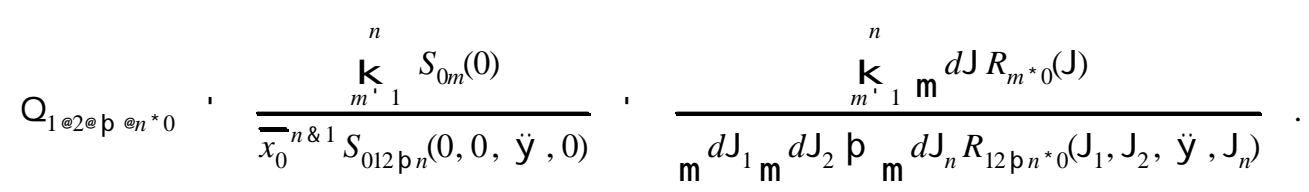

In so doing, it is prudent to ask, "How will these poly-spectral ratios depend upon reactivity?" It can and subsequently will be shown that, for extremely subcritical fissile systems, the $n$ th-order poly-spectral ratio will asymptotically approach

$$
\mathrm{Q}_{1 @ @ @ *_{0}} \operatorname{xx}_{\mathrm{D} 6 \& 4} \frac{\bar{\zeta}^{n}}{\bar{\delta}\left(\zeta_{0} \& 1\right) .\left(\delta_{\delta} \& n \% 1\right)} .
$$

On the other hand, for fissile systems approaching prompt critical, the $n$ th-order poly-spectral ratio will asymptotically behave as

$$
\mathrm{Q}_{1 @ @ @ * 0} \underset{\mathrm{D} 60}{\operatorname{xxxV}}{ }_{n} C_{2}\left(\frac{\overline{\bar{\delta}_{0}<* \mathrm{D} *}}{\overline{<(<\& 1)}}\right)^{n \& 1} .
$$

Monte Carlo calculational models of ${ }^{252} \mathrm{Cf}$-driven active measurements of simple uranium-metal systems have shown that the transition between these asymptotic behaviors in the trispectral (i.e., third-order) ratio occurs in the same region, $k-0.5$, as the transition observed in the bispectral ratio.[20] It is therefore reasonable to speculate that yet higher order spectral ratios will behave similarly.

The upper asymptotic behavior (102) of the poly-spectral ratios occurs due to a fundamental, and in retrospect obvious, property of higher order statistics. Successively higher order statistics are progressively more sensitive to reactivity. Recall 
that among the possible sequences of events that lead to an $n$-tuplet of correlated counts is a minimal sequence that contributes to the $n$ th-order poly-spectrum as

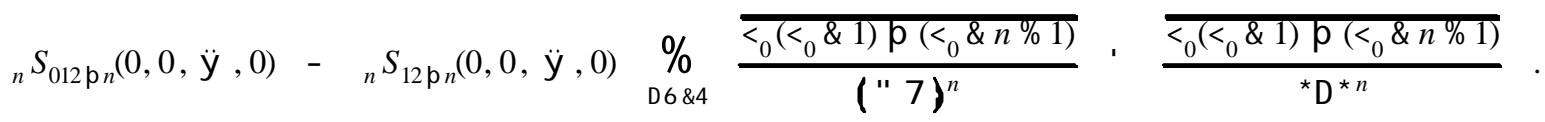

This sequence of events occurs when a source emits at least $n$ neutrons that successfully initiate at least $n$ branches in the chain-reaction. This is the shortest sequence of events. In extremely subcritical systems, it is also the most probable sequence of events, and it has the minimum sensitivity to reactivity characteristic of a given $n$ th-order statistic. Further recall that for a given order $n$, there is also a characteristic maximal sequence that contributes to the poly-spectrum as

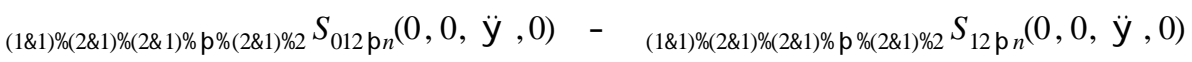

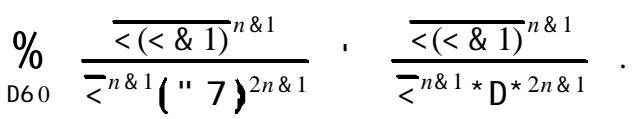

This sequence of events occurs when a source emits neutrons that themselves successfully initiate only one branch in the chain-reaction. However, this first branch is followed by a succession of induced fissions that each initiate two branches in the chain-reaction, such that by its terminus the chain-reaction has branched $2 n \& 1$ times. This is the longest sequence of events, and it is also the most probable sequence of events in fissile systems approaching prompt critical.

All other possible sequences of events leading to an $n$-tuplet of correlated counts will have sensitivity to reactivity somewhere between the lower and upper bounds (103) and (104). Monte Carlo calculational models of ${ }^{252} \mathrm{Cf}$ active source measurements of simple uranium-metal systems have produced results that adhere closely to these conclusions.[20]

Finally, observe that both the upper and lower asymptotic sequences possess progressively higher sensitivity to reactivity for successively higher order statistics. This feature of the higher order statistics is the one that promises to have the greatest potential utility. It can be applied to detect progressively smaller changes in subcritical reactivity that occur due to changes in composition, configuration, and fissile mass. Therefore, it may prove useful in systems identification applications since it should be able to distinguish between very similar, but still different, fissile assemblies. It is expected that this technique may be of particular interest to experimenters developing methods to support nuclear materials control and accountability.

\section{CONCLUSIONS}

A probabilistic technique has been used to develop theoretical models of high order statistics acquired from measurements of the temporal evolution of fission chain-reactions. The models developed encompass statistics acquired from both active and passive measurements of fissile systems, and they describe the temporal distribution of $n$-tuplets of correlated detector counts that in turn may be collectively correlated to active source emissions. This development unifies the Rossi- $\alpha$, pulsed neutron, and neutron noise analysis methods and generalizes them from a single method to measure pairs of correlated events to a single method to measure collections of an arbitrary number of correlated events.

It has been demonstrated that successively higher order statistics possess progressively higher sensitivity to reactivity. Consequently, these counting statistics are more sensitive to changes in the composition, configuration, and fissile mass of the system measured. Furthermore, it has been shown that this technique is capable of distinguishing the response of the fissile system to the active source from its response to intrinsic or inherent sources. It may in fact allow the measurement of properties of intrinsic sources that are otherwise difficult or impossible to observe directly. This ability in conjunction with their enhanced sensitivity indicates that these statistics may be of significant utility in a variety of applications. Potential applications include enhanced radiation signature identification of weapons components to support nuclear disarmament and safeguards efforts and augmented nondestructive analysis of spent nuclear fuel. In general, the technique should expand present capabilities in the analysis of neutron counting measurements. 


\section{ACKNOWLEDGMENTS}

I am indebted to John Mihalczo for suggesting this course of research to me and for complementing my predilection for deductive reasoning with his for the intuitive kind. I also owe Tim Valentine thanks for his advice and direction in deciphering previous developments, particularly those of Gordon E. Hansen, in order to acquire a solid foundation for the theoretical models described herein.

\section{REFERENCES}

[1] J. T. Mihalczo, "Use of ${ }^{252} \mathrm{Cf}$ as a Randomly Pulsed Neutron Source for Prompt Neutron Decay Measurements," YDR-41, Oak Ridge Y-12 Plant (1970).

[2] E. Rhodes, C. E. Dickerman, A. DeVolpi, and C. W. Peters, "APSTNG: Radiation Interrogation for Verification of Chemical and Nuclear Weapons," IEEE Trans. Nucl. Sci. 39, 1041 (1992).

[3] J.-L. Muñoz-Cobo, R. B. Pérez, and G. Verdú, "Stochastic Neutron Transport Theory: Neutron Counting Statistics in Nuclear Assemblies," Nucl. Sci. Eng. 95, 83 (1987).

[4] F. C. Difilippo, "Stochastic Processes in a Subcritical Nuclear Reactor in the Presence of a Fission Source," Nucl. Sci. Eng. 90, 13 (1985).

[5] R. Feynman, F. deHoffmann, and R. Serber, "Dispersion of the Neutron Emission in ${ }^{23}$ U Fission," J. Nucl. Energy 3, 64 (1956).

[6] J. D. Orndoff, "Prompt Neutron Periods of Metal Critical Assemblies," Nucl. Sci. Eng. 2, 450 (1957).

[7] G. E. Hansen, H. H. Helmick, and J. D. Orndoff, "Neutron Counting Statistics in Basic Fast Critical Assemblies," Proc. Joint Japan-United States Seminar on Reactor Noise Analysis (1968).

[8] M. Edelmann, "New Rossi- $\alpha$-Measurement Methods," INR-4/68-15, Karlsruhe Nuclear Research Center (1968).

[9] B. E. Simmons and J. S. King, “A Pulsed Neutron Technique for Reactivity Determination,” Nucl. Sci. Eng. 3, 595 (1958).

[10] J. T. Mihalczo and V. K. Paré, "Theory of Correlation Measurements in Time and Frequency Domains with ${ }^{25} \mathrm{Cf}$," ORNL/TM-4732, Oak Ridge National Laboratory (1974).

[11] W. Seifritz, D. Stegemann, and W. Väth, "Two-Detector Crosscorrelation Experiments in the Fast-Thermal Argonaut Reactor STARK," Intl. Symposium on Neutron Noise, Wave, and Pulse Propagation (1966).

[12] Tsutomo Nomura, Seiichiro Gotoh, and Kozo Yamaki, "Reactivity Measurements by Neutron Noise Analysis Using TwoDetector Correlation Method and Supercritical Reactor Noise Analysis,” Nippon Atomic Industry Group Co., Ltd.

[13] J. S. Bendat and A. G. Piersol, Random Data: Analysis and Measurement Procedures, $2^{\text {nd }}$ ed., John Wiley and Sons (1986).

[14] G. R. Cooper and C. D. McGillem, Probabilistic Methods of Signal and System Analysis, $2^{\text {nd }}$ ed., Harcourt-BraceJovanovich (1986).

[15] D. F. Elliot and K. R. Rao, Fast Transforms: Algorithms, Analyses, Applications, Academic Press (1982). 
[16] Modular Pulse-Processing Electronics and Semiconductor Radiation Detectors, EG\&G ORTEC (1997).

[17] J. M. Mendel, "Tutorial on Higher-Order Statistics (Spectra) in Signal Processing and System Theory: Theoretical Results and Some Applications," Proc. IEEE 79(3), 278 (1991).

[18] A. Papoulis, Probability, Random Variables, and Stochastic Processes, $2^{\text {nd }}$ ed., McGraw-Hill (1984).

[19] R. V. Meghreblian and D. K. Holmes, Reactor Analysis, McGraw-Hill (1960).

[20] J. K. Mattingly, High Order Statistical Signatures from Source-Driven Measurements of Subcritical Fissile Systems, Ph.D. Dissertation, University of Tennessee (1998). 


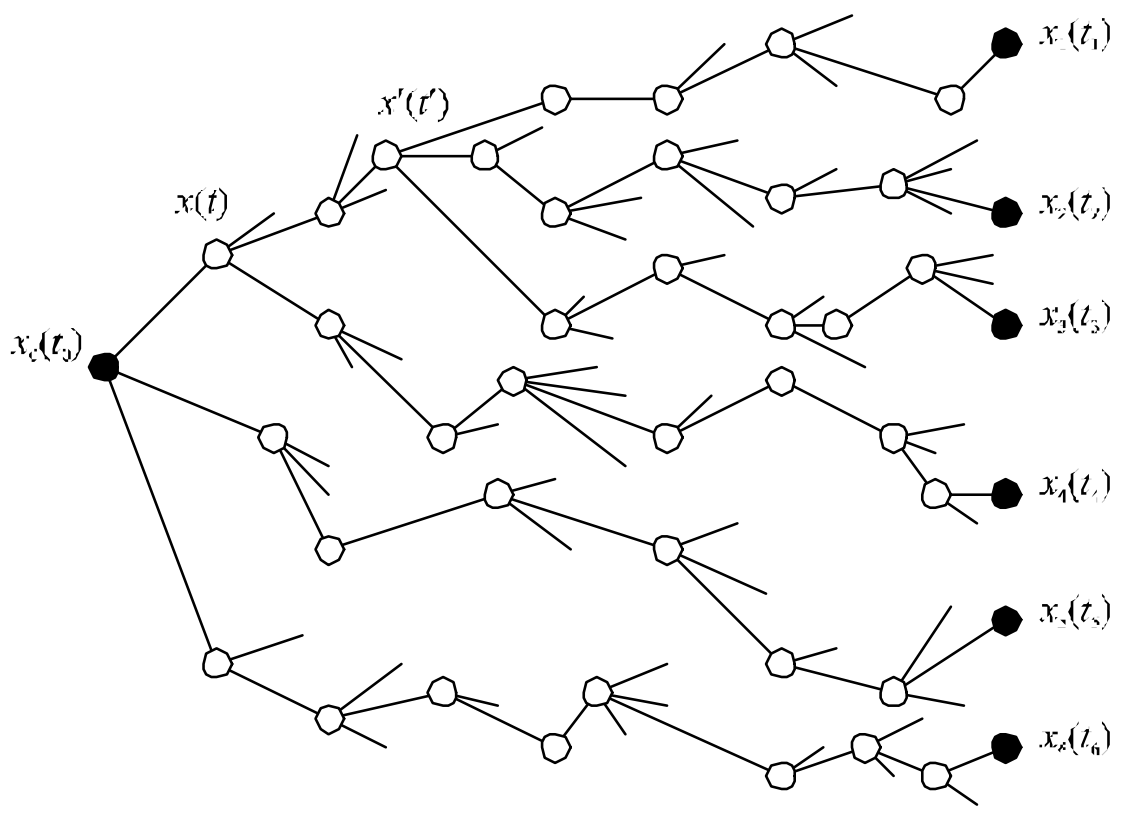

$t_{1}<t<t^{\prime \prime}<t \leq t_{2} \leq t_{3} \leq t \leq t \leq t_{2}$

Figure 3. One possible chain-reaction that can occur during an active measurement of a fissile system; closed circles denote observable events including detector counts and detected source emissions, open circles denote unobservable induced fission events, and each line indicates a neutron.

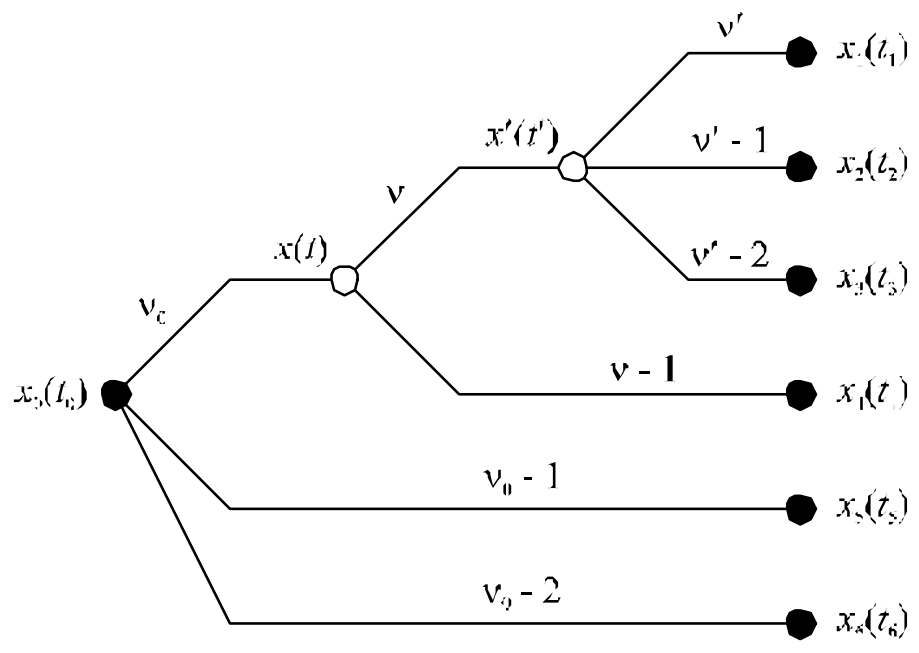

Figure 4. Diagram representing chain-reaction shown in Fig. 1; each line now indicates a series of "chain-related" neutrons, and the label associated with each line indicates the number of neutrons available to sustain that branch in the chain-reaction. 


\section{$x_{0}\left(t_{0}\right) \bullet \quad v_{0} \bullet x_{1}\left(t_{1}\right)$}

Figure 5. Diagram representing a chain-reaction terminating in one detector count (see also Fig. 2).
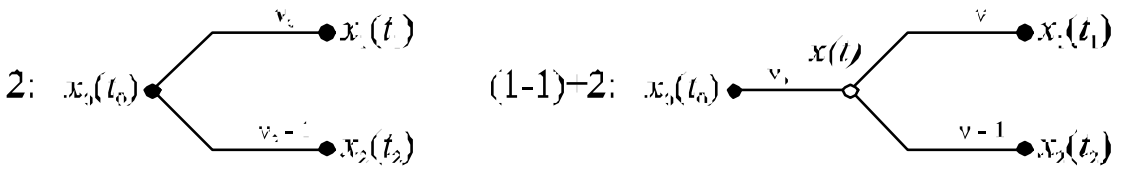

Figure 6. Diagram representing chain-reactions terminating in two correlated detector counts; the notation indicating each process denotes the neutron economy of the chain-reaction (see also Fig. 2).
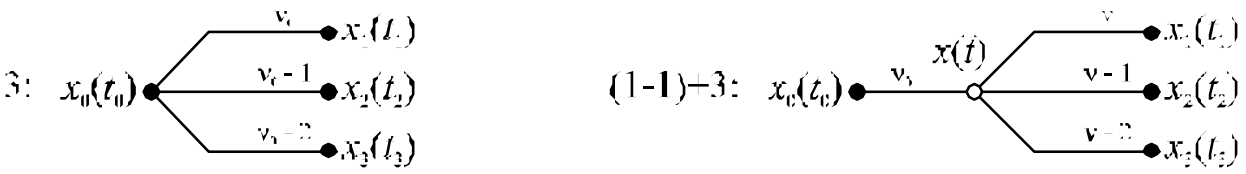

$(2-1)+2$ :
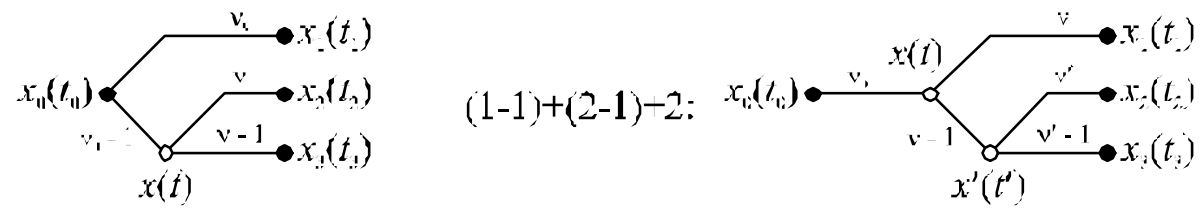

Figure 7. Diagram representing chain-reactions terminating in three correlated detector counts; the notation indicating each process again denotes the neutron economy of the chain-reaction (see also Fig. 2). 


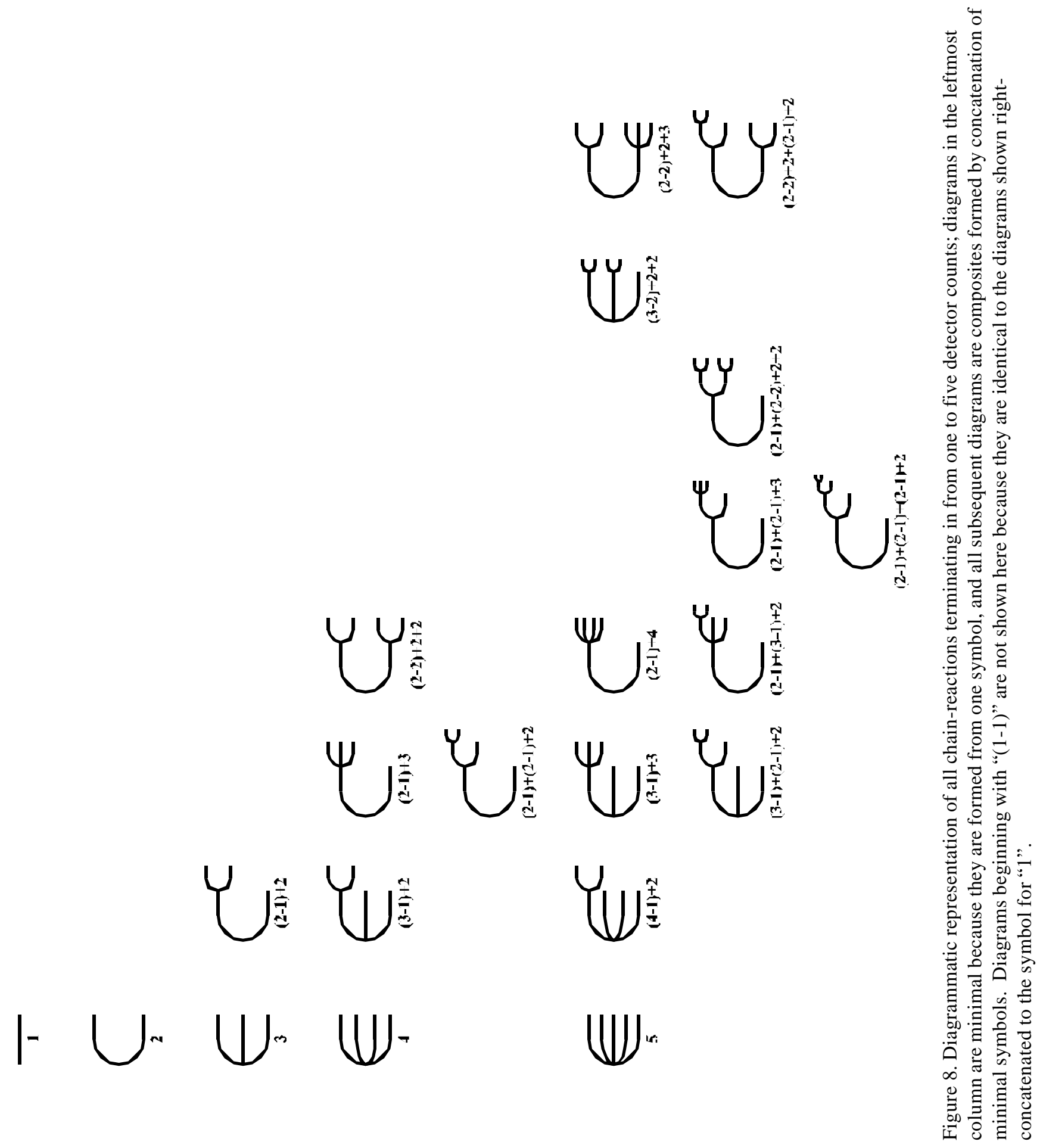


Table 1. Recursive expansions to generate all chain-reactions terminating in from one to six detector counts; notation indicates the neutron economy of each specific chain-reaction, the order indicates the number of detector counts, and the number of stages indicates the length of generated composite diagram formed from concatenating minimal symbols (see also Fig. 6).

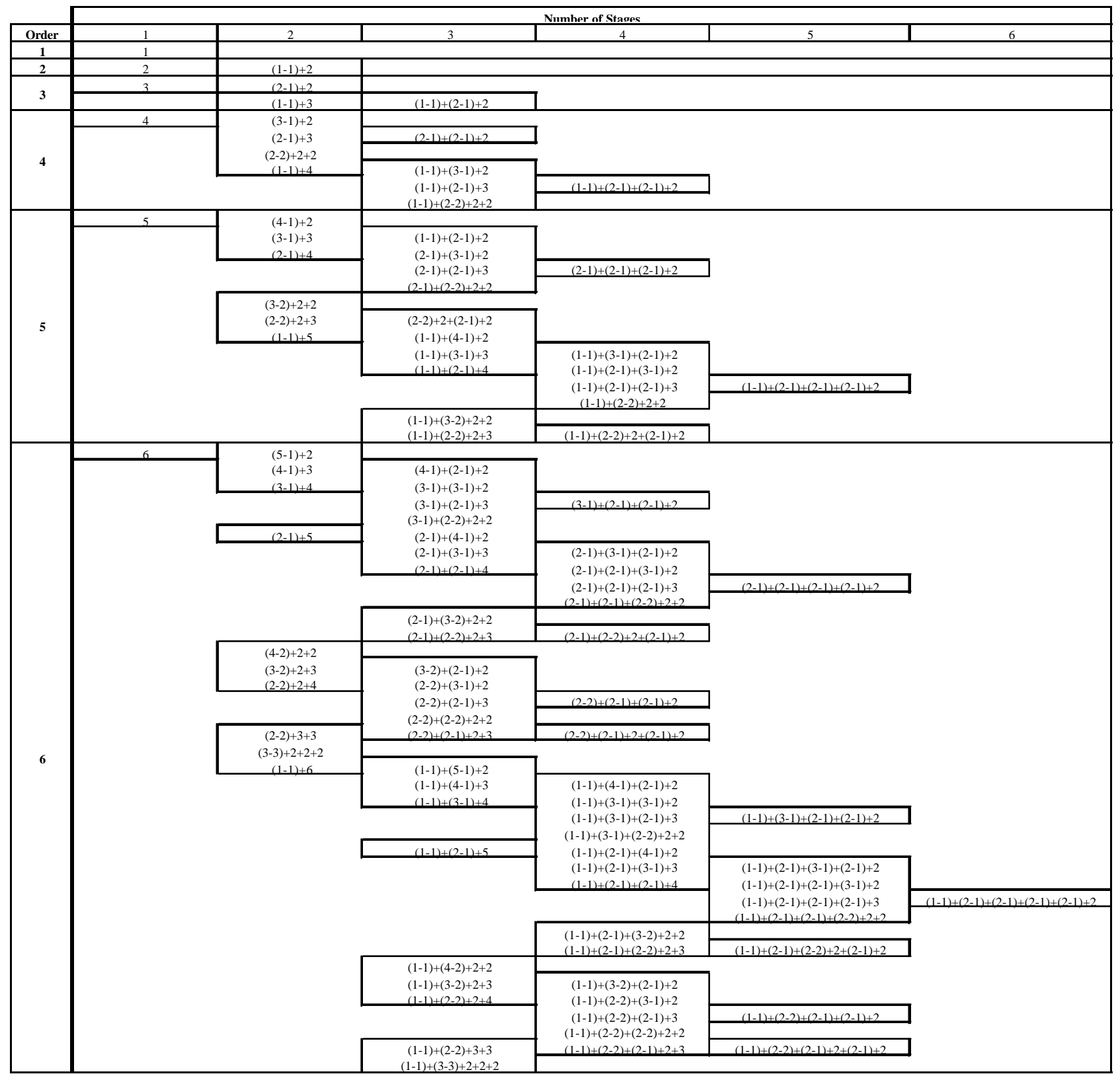




\section{DISTRIBUTION}

University of Tennessee

L. F. Miller

B. Upadhyaya

UT-Battelle, LLC.

L. G. Chiang

J. T. Mihalczo

J. A. Mullens

R. B. Oberer 